TRANSACTIONS OF THE

AMERICAN MATHEMATICAL SOCIETY

Volume 361, Number 8, August 2009, Pages 4091-4124

S 0002-9947(09)04649-2

Article electronically published on March 16, 2009

\title{
THE GEOGRAPHY PROBLEM FOR 4-MANIFOLDS WITH SPECIFIED FUNDAMENTAL GROUP
}

\author{
PAUL KIRK AND CHARLES LIVINGSTON
}

\begin{abstract}
For any class $\mathcal{M}$ of 4 -manifolds, for instance the class $\mathcal{M}(G)$ of closed oriented manifolds with $\pi_{1}(M) \cong G$ for a fixed group $G$, the geography of $\mathcal{M}$ is the set of integer pairs $\{(\sigma(M), \chi(M)) \mid M \in \mathcal{M}\}$, where $\sigma$ and $\chi$ denote the signature and Euler characteristic. This paper explores general properties of the geography of $\mathcal{M}(G)$ and undertakes an extended study of $\mathcal{M}\left(\mathbf{Z}^{n}\right)$.
\end{abstract}

\section{INTRODUCTION}

This paper addresses the following general question: in what ways does the fundamental group of a 4-manifold constrain its other algebraic invariants? To make this more precise, let $\mathcal{M}(G)$ denote the class of closed oriented topological 4-manifolds $M^{4}$ with $\pi_{1}\left(M^{4}\right) \cong G$. The geography problem for a group $G$ asks for the determination of the set $\mathcal{G}(G)=\{(\sigma(M), \chi(M)) \mid M \in \mathcal{M}(G)\}$, where $\sigma$ and $\chi$ denote the signature and Euler characteristic. Surprisingly, for most classes of groups, including free abelian groups, the geography is poorly understood.

Results that form the starting point for the study of the geography problem include that of: Winkelnkemper [34, who proved that if $G$ is abelian, then for any $M \in \mathcal{M}(G),|\sigma(M)| \leq \chi(M)$; Hausmann and Weinberger [11, who introduced and studied the function $q(G)=\min \{\chi(M) \mid M \in \mathcal{M}(G)\}$; and Kotschick 20, who began the investigation of the function $p(G)=\min \{\chi(M)-\sigma(G) \mid M \in \mathcal{M}(G)\}$. Also noteworthy is Gromov's article 8, which introduced the use of $L^{2}$-homology into the study of geography. Further related articles include [3, 4, 15, 16, 20, 23.

To simplify our exposition, we will restrict our work to the topological category. However, all our results apply identically in the smooth category. It is unknown whether the geography of a group depends on the category, though we will identify groups for which distinctions are likely to appear. The geography problem for a nontrivial fundamental group has also been studied extensively for the symplectic category in which far stronger constraints appear; references include [1, 17, 21].

In addition to presenting a general exposition of the topic and developing techniques for studying the geography problem, there are four main topics of this paper.

Received by the editors May 25, 2007.

2000 Mathematics Subject Classification. Primary 57M05, 57N13, 57R19.

Key words and phrases. Hausmann-Weinberger invariant, fundamental group, four-manifold, minimal Euler characteristic, geography.

This work was supported by grants from the NSF. 
The homological refinement. A 4-manifold $M \in \mathcal{M}(G)$ naturally determines a homology class, $[M] \in H_{4}(G)$, as well as a subspace $I(M)=$ image $H^{2}(G) \rightarrow$ $H^{2}(M)$. Thus, the geography problem can be refined by considering $\mathcal{M}(G, \alpha)=$ $\left\{M \mid \pi(M) \cong G\right.$ and $\left.[M]=\alpha \in H_{4}(G)\right\}$.

In Section 2 we show that the restriction of the intersection form of $M$ to $I(M)$ is determined by $\alpha$. When $M$ is aspherical, $I(M)=H^{2}(M)$, and when $M$ is simply connected $I(M)=0$. In general $I(M)$ and $\alpha$ capture that part of the intersection form determined by $G$. As we will describe in Section 2, every homology class $\alpha$ is represented by a smooth manifold, and smooth manifolds $M_{1}, M_{2} \in \mathcal{M}(G)$ represent the same class $\alpha$ if and only if they are stably diffeomorphic, where stabilization refers to a connected sum with $\pm \mathbf{C P}^{2}$. Clearly $\mathcal{G}(G)$ is the union of the sets $\mathcal{G}(G, \alpha)$.

Our first goal is to extend the techniques of earlier work to develop general properties of $\mathcal{G}(G, \alpha)$. Although we are able to find $\mathcal{G}(G, \alpha)$ for a variety of pairs $(G, \alpha)$, there is no case in which $\alpha$ is not primitive for which $\mathcal{G}(G, \alpha)$ is fully known.

Kotschick 20] briefly considered the case in which $\alpha=0$. In generalizing this, we explore how understanding the cohomology ring of $G$ reveals details in the structure of $\mathcal{G}(G)$.

The group $\mathbf{Z}^{n}$. In our earlier work on the geography problem [19] we gave an exact computation of the Hausmann-Weinberger function for all free abelian groups, $q\left(\mathbf{Z}^{n}\right)$. Here we undertake the study of $\mathcal{G}\left(\mathbf{Z}^{n}\right)$ and $\mathcal{G}\left(\mathbf{Z}^{n}, \alpha\right)$. Although we are not able to fully compute these sets in general, among our results is a complete description of $\mathcal{G}\left(\mathbf{Z}^{n}\right)$ for $n \leq 6$ and $\mathcal{G}\left(\mathbf{Z}^{n}, \alpha\right)$ for $n \leq 6$ and for primitive $\alpha$.

Asymptotics of Kotschick's function, $p\left(\mathbf{Z}^{n}\right)$. Prior to 19 the only known bounds on $q\left(\mathbf{Z}^{n}\right)$ were roughly $n^{2} / 2 \leq q\left(\mathbf{Z}^{n}\right) \leq n^{2}$. In 19 it was shown that in fact $q\left(\mathbf{Z}^{n}\right)$ is roughly $n^{2} / 2$. (Stated in terms of asymptotics, $q\left(\mathbf{Z}^{n}\right) / n^{2} \rightarrow 1 / 2$.) The dearth of any examples of manifolds $M$ for which the signature is large when compared to the second Betti number suggested that the Kotschick function might satisfy a similar growth pattern: $p\left(\mathbf{Z}^{n}\right) / n^{2} \rightarrow 1 / 2$. In Section 6 we disprove this, producing examples that show that $\lim \inf p\left(\mathbf{Z}^{n}\right) / n^{2}<1 / 2$.

Questions. The final section of this paper presents a discussion of open questions. Some of these focus on issues of category: primarily topological versus smooth, but we also note the essential appearance of another category, that of 4-dimensional Poincare complexes. Another focus is on the dependence of the geography on the homology class $\alpha$; for instance, whether $\mathcal{G}(G, \alpha)=\mathcal{G}(G,-\alpha)$ for all $G$ and $\alpha$ is unknown. Related to this is the role of orientation in geography. Another focus of questions is that of understanding the geography in terms of the structure of the group: this appears in the discussion of $\mathbf{Z}^{n}$; we also mention some unexpected issues that arise concerning free products.

Outline of the paper. In Section 2 we present the notation and basic tools of the geography problem. This includes a quick review of elementary topics such as simple properties of the Euler characteristic of 4-manifolds, general properties of symmetric bilinear forms, and the intersection forms of 4-manifolds. We also consider somewhat deeper topics, such as group homology and $G$-bordism theory as it applies to 4 -manifolds, proving for instance that $\mathcal{M}(G, \alpha)$ is nonempty for all finitely presented groups $G$ and homology classes $\alpha \in H_{4}(G)$. 
Section 3 reviews some of the past results related to the geography problem, stating them in a common notation, identifying their relationship to the general geography problem, and extending them to the homologically restricted problem of understanding $\mathcal{G}(G, \alpha)$. The section also completes the foundational work on $\mathcal{G}(G, \alpha)$.

Section 4 presents some basic examples of geography. Kotschick [20] observed that if $H_{4}(G)=0$, one can obtain bounds on $p(G)$. His observation extends in a relatively straightforward way to give constraints on the geography of $\mathcal{G}(G, \alpha)$ when $\alpha$ represents torsion in $H_{4}(G)$. Coupling this with explicit constructions of 4-manifolds, we completely determine $\mathcal{G}(G)$ when $G$ is a 2 -manifold or 3-manifold group.

In Section 5 we take on the most detailed analysis of the paper, focusing on the geography of $\mathbf{Z}^{n}$. The results for $\mathcal{G}\left(\mathbf{Z}^{n}\right)$ are complete for $n \leq 6$; and for $n=8$ we identify the first case in which there is a strong potential that the smooth category is distinct from the topological setting. The results for $\mathcal{G}\left(\mathbf{Z}^{n}, \alpha\right)$ are less complete, but we achieve a full analysis of $\mathcal{G}\left(\mathbf{Z}^{6}, \alpha\right)$ for all primitive classes $\alpha$.

Complementing the work on $\mathcal{G}\left(\mathbf{Z}^{n}\right)$ for $n$ small, as described in Section 5. Section 6 presents results concerning $\mathcal{G}\left(\mathbf{Z}^{n}\right)$ for $n$ large. Here we offer a new construction of 4-manifolds in $\mathcal{M}\left(\mathbf{Z}^{n}\right)$ for arbitrarily large values of $n$. These manifolds are sufficient to reveal unexpected sporadic behavior in the geography. To be somewhat more specific, for small $n$, any point in $\mathcal{G}\left(\mathbf{Z}^{n}\right)$ can be realized by a manifold constructed from a manifold of signature 0 by stabilization via connected sums with $\pm \mathbf{C P}^{2}$. In contrast, for appropriate large $n$, there are arbitrarily many elements of $\mathcal{G}\left(\mathbf{Z}^{n}\right)$ of large signature which cannot be realized via stabilizations of manifolds of smaller signature. These examples are used to study the asympotics of Kotschick's function, $p\left(\mathbf{Z}^{n}\right)$.

Section 7 discusses open problems in the study of the geography of 4-manifold groups.

\section{Notation AND BASiC Results}

In the interest of exposition, we have chosen to work in the category of topological manifolds. All of the work, however, applies identically in the smooth setting. There are many other possible categories of 4-manifolds, such as symplectic 4-manifolds, compact Kähler surfaces, irreducible or minimal 4-manifolds. Each choice leads to an interesting set of questions. Moreover, delicate issues arise when comparing the problems for different categories. One can also leave the realm of manifolds and consider 4-dimensional Poincaré complexes. Most of our discussion applies in this setting, and we anticipate that to fully understand the case of topological 4manifolds, the case of 4-dimensional Poincaré complexes will need to be analyzed so that surgery theory [6] might be applied. To be more precise, all the manifolds we construct will be smooth, and all our obstructions apply to 4-dimensional Poincaré complexes. At this time, there are no known pairs $(\sigma, \chi)$ that are known to be the signature and Euler characteristic of a 4-dimensional Poincaré complex but not for a smooth 4-dimensional manifold (for any choice of fundamental group $G$ ).

2.1. Euler characteristic. For a space $X$ we denote the integral and rational homology by $H_{*}(X)$ and $H_{*}(X ; \mathbf{Q})$, respectively. The Betti numbers are defined by $\beta_{i}(X)=\operatorname{dim}\left(H_{i}(X ; \mathbf{Q})\right)$. If the homology is finitely generated, the Euler characteristic is given by $\chi(X)=\sum(-1)^{i} \beta_{i}(X)$. For a closed, orientable 4-manifold $M$, 
Poincaré duality implies that

$$
\chi(M)=2-2 \beta_{1}(M)+\beta_{2}(M) .
$$

2.2. Symmetric bilinear forms and the signature. For convenience we recall some terminology and facts about symmetric bilinear integer forms.

We will be considering symmetric bilinear forms $F: V \times V \rightarrow \mathbf{Z}$ with $V$ a finitely generated free abelian group whose rank is called the rank of $F$. If the determinant of $F$ (in some basis) is \pm 1 the form is called unimodular; if the determinant is non-zero the form is called nondegenerate. If $F(v, v)$ is even for all $v \in V$, the form is called even, and otherwise it is called odd. The signature of $F$ is the difference

$$
\sigma(F)=b^{+}(F)-b^{-}(F),
$$

where $b^{+}(F)$ (respectively $b^{-}(F)$ ) is the dimension of the largest subspace of $V \otimes \mathbf{R}$ on which (the obvious extension of) $F$ is positive (respectively negative) definite. A nondegenerate form is called definite if $|\sigma(F)|$ equals the rank of $F$, and called indefinite otherwise.

Two basic unimodular (integral) forms are the indefinite even form of rank two and signature 0 , denoted $H$ (represented by a $2 \times 2$ matrix with diagonal entries 0 and off-diagonal entries 1) and the even positive definite form of rank 8 (and signature 8), denoted $E_{8}$.

The classification theorem (see for example [26]) states that any unimodular indefinite odd form is equivalent to a diagonal form with all diagonal entries \pm 1 , and thus is determined by its rank and signature. Any unimodular indefinite even form is a direct sum $k H \oplus m E_{8}$, where $k$ is a positive integer, and $m$ is an integer, and thus is again determined by its rank and signature. The classification of definite forms is not complete; all such even forms have rank and signature divisible by 8 .

An isotropic subspace of a symmetric bilinear form $F: V \times V \rightarrow \mathbf{Z}$ is a subspace $W$ so that $F\left(w_{1}, w_{2}\right)=0$ for all $w_{1}, w_{2} \in W$. If $F$ is nondegenerate and admits an $n$-dimensional isotropic subspace, an easy argument shows that $b^{+}(F) \geq n$ and $b^{-}(F) \geq n$, so that

$$
\operatorname{rank}(F) \geq|\sigma(F)|+2 n \text {. }
$$

2.3. Algebraic topology and bordism results. For a finitely presented group $G$ there is an associated Eilenberg-Mac Lane space which we denote $B_{G}$. By definition, $B_{G}$ is a based, connected CW-complex whose fundamental group $\pi_{1}\left(B_{G}\right)$ is identified with $G$ and $\pi_{i}\left(B_{G}\right)=0, i \geq 2$; the homotopy type of $B_{G}$ is unique. The homology groups $H_{*}(G)$ and the cohomology ring $H^{*}(G)$ are defined to be $H_{*}\left(B_{G}\right)$ and $H^{*}\left(B_{G}\right)$, where coefficients are assumed to be integers unless specifically noted.

Every compact $m$-manifold has the homotopy type of a finite $\mathrm{CW}$-complex. (In fact, by results of Kirby-Siebenmann [18, every compact manifold $M$ has the homotopy type of a finite polyhedron.) Thus, given a homomorphism $\phi: \pi_{1}(M) \rightarrow$ $G$, there is a corresponding based map, unique up to based homotopy, $f: M \rightarrow B_{G}$ inducing $\phi$ on fundamental groups. This proves the following well-known lemma.

Lemma 2.1. Let $M$ be a closed, oriented $m$-manifold and $G$ a discrete group. A homomorphism $\phi: \pi_{1}(M) \rightarrow G$ uniquely determines an element $\alpha=f_{*}([M]) \in$ $H_{m}(G)$, where the map $f: M \rightarrow B_{G}$ is the unique based homotopy class inducing $\phi$ and $[M] \in H_{m}(M)$ denotes the orientation class. 
Thus we will refer to $\alpha \in H_{m}(G)$ as the class induced by $\phi: \pi_{1}(M) \rightarrow G$.

Bilinear pairings can be constructed on the cohomology $H^{*}(G)$ using the cup and cap products as follows. Given a class $\alpha \in H_{2 n}(G)$, define

$$
H^{n}(G) \times H^{n}(G) \rightarrow \mathbf{Z} \text { by }(x, y) \mapsto(x \cup y) \cap \alpha .
$$

When $n$ is even, the pairing (2.3) is symmetric.

Definition 2.2. Suppose that $M$ is an oriented closed $2 n$-dimensional manifold and $\phi: \pi_{1}(M) \rightarrow G$ is a homomorphism, with $f: M \rightarrow B_{G}$ the corresponding homotopy class of maps and $\alpha=f_{*}([M]) \in H_{2 n}(G)$ the class induced by $\phi$.

Define the subspace

$$
\begin{aligned}
I(M, f)=\text { image }\left(f^{*}: H^{n}(G) \rightarrow H^{n}(M) \rightarrow\right. & \left.H^{n}(M) / \text { torsion }\right) \\
& \subset H^{n}(M) / \text { torsion. }
\end{aligned}
$$

The bilinear pairing (2.3) completely determines the restriction of the intersection form of $M$,

$$
H^{n}(M) / \text { torsion } \times H^{n}(M) / \text { torsion } \rightarrow \mathbf{Z},(x, y) \mapsto(x \cup y) \cap[M],
$$

to $I(M, f)$, since

$$
\left(f^{*}(a) \cup f^{*}(b)\right) \cap[M]=(a \cup b) \cap \alpha .
$$

In particular, if $f^{*}: H^{n}(G) \rightarrow H^{n}(M)$ is surjective, then the intersection form of $M$ is completely determined by algebra, or, more precisely, by the cohomology ring $H^{*}(G)$ and the class $\alpha \in H_{2 n}(G)$.

The problem of deciding when a class $\alpha \in H_{m}(G)$ is represented by a map $f: M \rightarrow B_{G}$ from a closed $m$-manifold is a classical (and difficult) problem in topology (see for example [30]), but can always be solved for $m=4$. We outline the argument.

Lemma 2.3. Given any group $G$ and class $\alpha \in H_{4}(G)$, there exists an oriented closed smooth 4-manifold $M$ and a continuous map $f: M \rightarrow B_{G}$ so that $f_{*}([M])=$ $\alpha$.

Proof. The proof of this follows from a calculation using the bordism spectral sequence, which is itself an application of the Atiyah-Hirzebruch spectral sequence (see 28] or [32]) applied to the generalized homology theory given by oriented bordism, $\Omega_{*}^{S O}$. In greater detail, there is a spectral sequence, with $E_{2}$-term given by $\left\{H_{i}\left(X, \Omega_{j}^{S O}\right)\right\}$, converging to $\Omega_{*}^{S O}(X)$. The relevant coefficient groups, oriented bordism groups of a point, are given by $\Omega_{0}^{S O}=\mathbf{Z}, \Omega_{1}^{S O}=0, \Omega_{2}^{S O}=0, \Omega_{3}^{S O}=0$, and $\Omega_{4}^{S O}=\mathbf{Z}$, generated by $\mathbf{C P}^{2}$ (see [27]). Thus, it follows from the spectral sequence that there is an exact sequence $\Omega_{4}^{S O} \rightarrow \Omega_{4}^{S O}(G) \rightarrow H_{4}(G, \mathbf{Z}) \rightarrow 0$, giving the desired surjection.

The problem of determining the size of $I(M, f)$ is also difficult in general, but the following fact gives a simple criterion when $M$ is a $4-$ manifold.

Proposition 2.4. Suppose that $f: M \rightarrow B_{G}$ induces an isomorphism $f_{*}: \pi_{1}(M) \rightarrow$ $G$. Then the homomorphism $f_{*}: H_{2}(M) \rightarrow H_{2}(G)$ is surjective and $f^{*}: H^{2}(G) \rightarrow$ $H^{2}(M)$ is injective. In particular, $\operatorname{rank}(I(M, f))=\beta_{2}(G)$. 
Proof. The Hopf exact sequence

$$
\pi_{2}(M) \rightarrow H_{2}(M) \stackrel{f_{*}}{\longrightarrow} H_{2}(G) \rightarrow 0
$$

implies that $f_{*}: H_{2}(M) \rightarrow H_{2}(G)$ is surjective. The result for cohomology follows from the universal coefficient theorem.

Surgery yields a method to ensure that a given class $\alpha \in H_{m}(G)$ is represented by a map $f: M^{m} \rightarrow B_{G}$ inducing an isomorphism on fundamental groups. The following argument is roughly that of Wall [31, Theorem 1.2], for the most part translated into combinatorial group theory.

Lemma 2.5. Suppose $G$ is finitely presented and $\alpha \in H_{m}(G), m>3$, is represented by $f: M \rightarrow B_{G}$ for a closed oriented $m$-manifold $M$. Then it is also represented by $g: N \rightarrow B_{G}$ so that the induced morphism $g_{*}: \pi_{1}(N) \rightarrow G$ is an isomorphism.

Proof. We first show that if $\phi: H \rightarrow G$ is a homomorphism of finitely presented groups, then $\phi$ can be extended to an epimorphism $\Phi: H * F \rightarrow G$ (where $H * F$ is the free product of $H$ with a finitely generated free group $F$ ) so that the kernel of $\Phi$ is normally generated by finitely many elements.

Indeed, given presentations

$$
H=\left\langle h_{1}, \cdots, h_{a} \mid w_{1}, \cdots, w_{b}\right\rangle \text { and } G=\left\langle g_{1}, \cdots, g_{c} \mid r_{1}, \cdots, r_{d}\right\rangle,
$$

let $F$ denote the free group generated by $g_{1}, \cdots, g_{c}$. Then

$$
H * F=\left\langle h_{1}, \cdots, h_{a}, g_{1}, \cdots, g_{c} \mid w_{1}, \cdots, w_{b}\right\rangle
$$

and there is an obvious epimorphism of $H * F$ to $G$, taking $h_{i}$ to $\phi\left(h_{i}\right)$ and $g_{i} \in F$ to $g_{i} \in G$. For $i=1, \cdots, a$, let $z_{i} \in F$ be a word in the $g_{i} \in F$ which is sent to $\phi\left(h_{i}\right) \in G$ by the canonical surjection $F \rightarrow G$. Taking the quotient of $H * F$ by the subgroup generated by the finitely many elements $r_{1}, \cdots, r_{d}, z_{1} h_{1}^{-1}, \cdots, z_{a} h_{a}^{-1}$ clearly yields $G$, since the generators $h_{i}$ and relations $z_{j} h_{j}^{-1}$ can be eliminated.

Thus by replacing $M$ by the connected sum of $M$ with finitely many copies of $S^{1} \times S^{m-1}$ we may arrange that $\pi_{1}(M) \rightarrow G$ is surjective and has kernel normally generated by finitely many elements. This can be done without changing the homology class $\alpha$, by arranging that the maps of $S^{1} \times S^{m-1}$ to $B_{G}$ factor through $S^{1}$.

Since $M$ is orientable and has dimension greater than three, the set of normal generators of the kernel can be represented by disjointly embedded circles with trivial normal bundles, and surgery on these circles yields a manifold $N$ with $\pi_{1}(N) \cong G$. Extending $M \rightarrow B_{G}$ to the trace of the surgery shows that $N$ maps to $B_{G}$, inducing an isomorphism on fundamental groups, without altering the class $\alpha \in H_{m}(G)$.

Given $\alpha \in H_{4}(G)$, denote by $\mathcal{M}(G, \alpha)$ the class of all pairs $(M, f)$, where $f: M \rightarrow$ $B_{G}$ induces an isomorphism $f_{*}: \pi_{1}(M) \rightarrow G$ and so that $\alpha=f_{*}([M])$. Then set $\mathcal{G}(G, \alpha)=\{(\sigma(M), \chi(M)) \mid(M, f) \in \mathcal{M}(G, \alpha)\}$. Thus $\mathcal{M}(G, \alpha)$ is nonempty for each $\alpha \in H_{4}(G)$ by Proposition 2.4 and Lemma 2.5. Furthermore, $\mathcal{G}(G)$ is the union of the $\mathcal{G}(G, \alpha)$. 
Corollary 2.6. Let $\alpha \in H_{4}(G)$ and let $(M, f) \in \mathcal{M}(G, \alpha)$. Then the restriction of the intersection form of $M$ to $I(M, f)$ depends only on $G$ and $\alpha \in H_{4}(G)$; it is given by the pairing (2.3): $(x, y) \mapsto(x \cup y) \cap \alpha$. Moreover,

(1) $b^{ \pm}(M) \geq b^{ \pm}(I(M, f))$,

(2) any isotropic subspace of the pairing (2.3) is also isotropic for the intersection form of $M$, and

(3) if (2.3) is even, then the restriction of the intersection form of $M$ to $I(M, f)$ is also even.

Thus if $I(M, f)=H^{2}(M) /$ torsion (for instance, if $f^{*}: H^{2}(G) \rightarrow H^{2}(M)$ is surjective), then the intersection form of $M$ is determined by $\alpha$.

2.4. Automorphisms of $G$. As usual, there are issues of basepoints that arise with the identification of $\pi_{1}(M)$ with a group $G$. This and other issues are resolved by considering the action of the automorphism group of $G$ on $H_{4}(G)$.

Proposition 2.7. Let $\Psi: G \rightarrow G$ be an automorphism of $G$, inducing an automorphism $\Psi_{*}: H_{*}(G) \rightarrow H_{*}(G)$. Then $\mathcal{G}(G, \alpha)=\mathcal{G}\left(G, \Psi_{*}(\alpha)\right)$. In other words, $\mathcal{G}(G, \alpha)$ depends only on the orbit of $\alpha$ under the action of $\operatorname{Aut}(G)$ on $H_{4}(G)$. The fundamental class of any oriented closed 4-manifold $M$ with $\pi_{1}(M)$ isomorphic to $G$ determines a well-defined element in the orbit set $H_{4}(G) / \operatorname{Aut}(G)$. Moreover, the subspace $I(M, f) \subset H^{2}(M) /$ torsion depends only on $M$ and not the map $f: M \rightarrow B_{G}$.

Proof. Any automorphism $\Psi: G \rightarrow G$ is induced by a based homotopy equivalence $h: B_{G} \rightarrow B_{G}$. Hence if $(M, f) \in \mathcal{M}(G, \alpha)$, then $(M, h \circ f) \in \mathcal{M}\left(G, \Psi_{*}(\alpha)\right)$ and so $\mathcal{G}(G, \alpha) \subset \mathcal{G}\left(G, \Psi_{*}(\alpha)\right)$. The reverse inclusion follows by considering $\Psi^{-1}$.

Given a manifold $M$ with $\pi_{1}(M)$ isomorphic to $G$, a choice of isomorphism determines a map $f: M \rightarrow B_{G}$ inducing the given isomorphism on fundamental groups. Another choice gives a possibly different map $g: M \rightarrow B_{G}$. The automorphism $g_{*} \circ f_{*}^{-1}: \pi_{1}\left(B_{G}\right) \rightarrow \pi_{1}\left(B_{G}\right)$ is induced by a based homotopy equivalence $\Psi: B_{G} \rightarrow B_{G}$. Thus $\Psi_{*}\left(f_{*}([M])\right)=g_{*}([M])$. Hence $f_{*}([M])$ is well defined in $H_{4}(G) / \operatorname{Aut}(G)$. Also the image $I(M, g)$ equals $I(M, \Psi \circ f)=I(M, f)$, since $\Psi^{*}: H^{2}(G) \rightarrow H^{2}(G)$ is an automorphism.

A consequence of Proposition 2.7 is that if we denote by $[\alpha] \in H_{4}(G) / \operatorname{Aut}(G)$ the orbit of $\alpha$, then it makes sense to define $\mathcal{M}(G,[\alpha])$ to be the class of 4 -manifolds $M$ whose fundamental group is isomorphic to $G$ such that $f_{*}([M]) \in[\alpha]$ for some (and hence any) reference map $f: M \rightarrow B_{G}$. In particular, $\mathcal{M}(G)$ is the disjoint union of the $\mathcal{M}(G,[\alpha])$, and the geography $\mathcal{G}(M,[\alpha])$ is well defined. Put another way, there is a well-defined surjective assignment $\mathcal{M}(G) \rightarrow H_{4}(G) / \operatorname{Aut}(G)$; this takes homotopy equivalent $M_{1}, M_{2} \in \mathcal{M}(G)$ to the same $[\alpha] \in H_{4}(G) / \operatorname{Aut}(G)$. Moreover, the subspace $I(M, f) \subset H^{2}(M) /$ torsion does not depend on the map $f$ when $f: M \rightarrow B_{G}$ induces an isomorphism on fundamental groups. Thus given $M \in \mathcal{M}(G,[\alpha])$, we simply use the notation $I(M)$ rather than $I(M, f)$ below.

Combined with Corollary 2.6 these observations imply that given any two manifolds $M_{1}, M_{2} \in \mathcal{M}(G,[\alpha])$, their intersection forms contain the isometric subforms $I\left(M_{1}\right)$ and $I\left(M_{2}\right)$ of rank $\beta_{2}(G)$. This bilinear form is the one given by Equation (2.3).

A simple bordism argument pointed out to the authors by Peter Teichner shows that two smooth manifolds $M_{1}$ and $M_{2}$ are in $\mathcal{M}(G,[\alpha])$ if and only if the connected 
sum of $M_{1}$ with sufficiently many copies of $\mathbf{C P}^{2}$ and $-\mathbf{C P}^{2}$ is diffeomorphic to the connected sum of $M_{2}$ with sufficiently many copies of $\mathbf{C P}^{2}$ and $-\mathbf{C} \mathbb{P}^{2}$.

The argument can be summarized as follows. Given $M_{1}, M_{2} \in \mathcal{G}(G,[\alpha])$, choose maps $f_{1}: M_{1} \rightarrow B_{G}$ and $f_{2}: M_{2} \rightarrow B_{G}$ such that $\left(f_{1}\right)_{*}\left(\left[M_{1}\right]\right)=\left(f_{2}\right)_{*}\left(\left[M_{2}\right]\right) \in$ $H_{4}(G)$. The bordism spectral sequence shows that after possibly replacing $M_{1}$ by its connected sum with some copies of $\pm \mathbf{C P}^{2}$, there is a bordism $F: W^{5} \rightarrow B_{G}$ from $\left(M_{1}, f_{1}\right)$ to $\left(M_{2}, f_{2}\right)$. By performing surgery on $W^{5}$, it can be arranged that $\pi_{1}\left(W^{5}\right) \cong G$ and that the inclusions of $M_{1}$ and $M_{2}$ are isomorphisms on $\pi_{1}$. The 1-handles of the bordism can be surgered without changing $\pi_{1}$ and similarly the the 4 -handles can be removed. The 2-handles are necessarily added along null homotopic, and thus null isotopic, curves. It follows that the portion of the bordism built from the 2-handles has, as its boundary components, $M_{1}$ and $M_{1} \#_{i} S_{i}$, where the $S_{i}$ are either $S^{2} \times S^{2}$ or $\mathbf{C P}^{2} \#\left(-\mathbf{C P}^{2}\right)$. Since $S_{i} \# \mathbf{C} \mathbb{P}^{2}$ is diffeomorphic to a connected sum of projective spaces, the desired result follows. (A related but much deeper argument applies in the topological category; there one might also need to take a connected sum with the Chern manifold, a nonsmoothable homotopy $\mathbf{C} \mathbb{P}^{2}$.)

Reversing orientation changes the sign of $\sigma$ and preserves $\chi$, and hence one obtains the following.

Proposition 2.8. For any $G, \mathcal{G}(G)$ is symmetric with respect to reflection through the $\chi$-axis. More generally, $\mathcal{G}(G,-\alpha)$ is obtained by reflecting $\mathcal{G}(G, \alpha)$ through the $\chi$-axis. Moreover, if $\alpha \in H_{4}(G)$ and $G$ admits an automorphism $\Psi$ so that $\Psi_{*}(\alpha)=-\alpha$, then $\mathcal{G}(G, \alpha)$ is symmetric with respect to the $\chi$-axis.

There is no reason why $\mathcal{G}(G, \alpha)$ should be symmetric for general $\alpha$. (The symmetry assertions in Proposition 2.8 extend to geography problems for classes of 4-manifolds closed under change of orientation; this excludes symplectic manifolds, for example.)

\section{Geography and Fundamental Groups}

3.1. Basic properties of $\mathcal{G}(G)$. We now apply the observations of the previous section to the problem of determining the geography associated to the class of 4 -manifolds with a specified fundamental group.

First, note that Equation (2.1) and the fact that $\beta_{1}(X)=\beta_{1}(G)$ for a connected space $X$ satisfying $\pi_{1}(X)=G$ imply that if $M$ is a closed, orientable 4-manifold with fundamental group $G$, then

$$
\chi(M)=2-2 \beta_{1}(G)+\beta_{2}(M) .
$$

We have the following basic observations.

Proposition 3.1. If $M$ is a closed 4-manifold and $\pi_{1}(M) \cong G$, then

(1) $\chi(M) \equiv \sigma(M)(\bmod 2)$.

(2) $\chi(M) \geq \beta_{2}(G)-2 \beta_{1}(G)+2$.

(3) $\chi(M) \geq|\sigma(M)|-2 \beta_{1}(G)+2$.

Proof. We have that $\beta_{2}(M)=b^{+}(M)+b^{-}(M)$ and $\sigma(M)=b^{+}(M)-b^{-}(M)$. The mod 2 congruence then follows from Equation (3.1).

Proposition 2.4 shows that $\beta_{2}(M) \geq \beta_{2}(G)$. Together with Equation (3.1) this proves the first inequality. 
Again, using that $\beta_{2}(M)=b^{+}(M)+b^{-}(M)$ and $\sigma(M)=b^{+}(M)-b^{-}(M)$, we have that

$$
\chi(M)=2-2 \beta_{1}(G) \mp \sigma(M)+2 b^{ \pm}(M) .
$$

The second inequality now follows from the fact that $b^{ \pm}(M) \geq 0$.

Proposition 3.1 gives us our first interesting constraint on the geography.

Corollary 3.2. Fix $G$ and $\alpha \in H_{4}(G)$. Then

$$
\mathcal{G}(G, \alpha) \subset \mathcal{G}(G)
$$

and $\mathcal{G}(G)$ is a subset of the intersection of the three half-planes

$$
\begin{gathered}
\mathcal{G}(G) \subset\left\{\chi \geq \beta_{2}(G)-2 \beta_{1}(G)+2\right\} \cap\left\{\chi+\sigma \geq-2 \beta_{1}(G)+2\right\} \cap\left\{\chi-\sigma \geq-2 \beta_{1}(G)+2\right\} . \\
\text { Moreover, if }(\sigma, \chi) \in \mathcal{G}(G, \alpha) \text {, then }(\sigma+1, \chi+1),(\sigma-1, \chi+1),(\sigma, \chi+2) \in \mathcal{G}(G, \alpha) .
\end{gathered}
$$

Proof. All but the last assertion follow from Proposition 3.1. For the last assertion, observe that a map $f: M \rightarrow B_{G}$ can be homotoped to be constant on a 4-ball in $M$. Construct a map on the connected sum $f^{\prime}: M \# \mathbf{C P}^{2} \rightarrow B_{G}$ by making $f^{\prime}$ constant on the $\mathbf{C P}^{2}$ factor. Since $\mathbf{C P}^{2}$ is simply connected, $f$ induces an isomorphism on fundamental groups. Moreover, $f^{\prime}$ and $f$ determine the same class $\alpha \in H_{4}(M)$ since $f^{\prime}$ is constant on $\mathbf{C P}^{2}$. Hence if $f: M \rightarrow B_{G}$ represents $(\sigma, \chi) \in \mathcal{G}(G, \alpha)$, $f^{\prime}: M \# \mathbf{C P}^{2} \rightarrow B_{G}$ represents $(\sigma+1, \chi+1) \in \mathcal{G}(G, \alpha)$. Similar arguments using $-\mathbf{C P}^{2}$ and $S^{2} \times S^{2}$ show that $(\sigma-1, \chi+1),(\sigma, \chi+2) \in \mathcal{G}(G, \alpha)$.

We will show below that these bounds are not strict in the case of $G \cong \mathbf{Z}^{n}, n \geq 2$. As an example, in Figure 1 we illustrate with three dark lines the bounds given by Corollary 3.2 for $G \cong \mathbf{Z}^{3}$ while the black dots indicate the only values that actually occur in the region, as will be shown later.

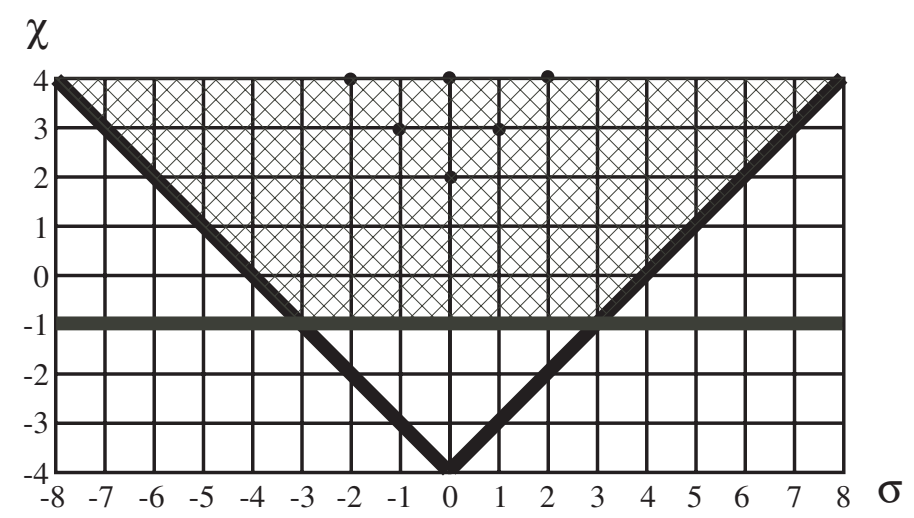

Figure 1. The bounds of Corollary 3.2 and the geography of $G \cong \mathbf{Z}^{3}$ 
3.2. The invariants of Hausmann-Weinberger and Kotschick. We now explain the relationship between the problem of identifying $\mathcal{G}(G)$ with previously studied invariants, notably the Hausmann-Weinberger invariant [1].

$$
q(G)=\inf \{\chi(M) \mid M \in \mathcal{M}(G)\}
$$

and its variant due to Kotschick 20,

$$
p(G)=\inf \{\chi(M)-\sigma(M) \mid M \in \mathcal{M}(G)\} .
$$

There are other equally interesting functions based on the geography. For instance, in complex geometry one considers the holomorphic Euler characteristic $\chi_{h}(M)$ and $c_{1}^{2}(M)$. Since $\chi_{h}(M)=\frac{1}{4}(\sigma(M)+\chi(M))$ and $c_{1}^{2}(M)=3 \sigma(M)+2 \chi(M)$ whenever these are defined, the pairs $\left(\chi_{h}, c_{1}^{2}\right)$ and $(\sigma, \chi)$ contain the same information. This led Baldridge-Kirk [1] to define the most general such function interpolating between the Hausmann-Weinberger function and the Kotschick function:

$$
f_{G}(t)=\inf \{\chi(M)+t \sigma(M) \mid M \in \mathcal{M}(G)\}, t \in \mathbf{R} .
$$

Note that $q(G)=f_{G}(0)$ and $p(G)=f_{G}(-1)$.

The Hausmann-Weinberger invariant can be viewed in the present context as the smallest $\chi$-intercept of any horizontal line which intersects $\mathcal{G}(G)$ nontrivially. Kotschick's variant is similar, but considers lines of slope 1 rather than slope 0 . More generally, $f_{G}(t)$ is the smallest $\chi$-intercept of a line of slope $-t$ that intersects $\mathcal{G}(G)$ nontrivially.

Corollary 3.2 shows that translating by $(0,2)$ maps $\mathcal{G}(G)$ and $\mathcal{G}(G, \alpha)$ into themselves. This motivates the introduction of the following functions.

\section{Definition 3.3.}

(1) $q_{G}(\sigma)=\min \{\chi(M) \mid M \in \mathcal{M}(G)$ and $\sigma(M)=\sigma\}$.

(2) $q_{G, \alpha}(\sigma)=\min \{\chi(M) \mid(M, f) \in \mathcal{M}(G, \alpha)$ and $\sigma(M)=\sigma\}$.

The basic properties of $q(G), p(G), f_{G}(t), q_{G}(\sigma)$ and $q_{G, \alpha}(\sigma)$ are summarized in the following theorem.

Theorem 3.4. For each finitely presented group $G$ and $\alpha \in H_{4}(G)$ :

(1) $q_{G}(\sigma)$ is defined for all $\sigma \in \mathbf{Z}$.

(2) $q_{G}(\sigma) \equiv \sigma \bmod 2$.

(3) $q_{G}(\sigma) \geq \max \left\{|\sigma|-2 \beta_{1}(G)+2, \beta_{2}(G)-2 \beta_{1}(G)+2\right\}$.

(4) $q_{G}(\sigma+1)=q_{G}(\sigma) \pm 1$.

(5) $\mathcal{G}(G)=\left\{(\chi, \sigma) \in \mathbf{Z}^{2} \mid \chi \geq q_{G}(\sigma)\right.$ and $\left.\chi \equiv \sigma(\bmod 2)\right\}$. Thus $q_{G}(\sigma)$ determines and is determined by $\mathcal{G}(G)$.

(6) For all large values of $\sigma, q_{G}(\sigma)=\sigma+p(G)$, where $p(G) \in \mathbf{Z}$ denotes Kotschick's invariant.

(7) The Hausmann-Weinberger invariant $q(G)$ equals $\min \left\{q_{G}(\sigma) \mid \sigma \in \mathbf{Z}\right\}$.

(8) $f_{G}(t) \neq-\infty$ if and only if $t \in[-1,1]$. The convex hull of $\mathcal{G}(G)$ in $\mathbf{R}^{2}$ is the intersection of the half-planes $\left\{\chi+t \sigma \geq f_{G}(t) \mid t \in[-1,1]\right\}$. Thus $f_{G}(t)$ determines and is determined by the convex hull of $\mathcal{G}(G)$.

(9) $q_{G}(\sigma) \leq q_{G, \alpha}(\sigma)$.

(10) $q_{G}(-\sigma)=q_{G}(\sigma)$ and $q_{G,-\alpha}(\sigma)=q_{G, \alpha}(-\sigma)$. 
The first 5 assertions hold for $q_{G, \alpha}$ also. Moreover,

$(6)^{\prime}$ There exist integers $p_{+, \alpha}(G)$ and $p_{-, \alpha}(G)$ (depending on $G$ and $\alpha$ ) satisfying $\left|p_{ \pm, \alpha}(G)\right| \geq p(G)$ and such that for all large values of $\sigma, q_{G, \alpha}(\sigma)=\sigma+$ $p_{+, \alpha}(G)$ and $q_{G, \alpha}(-\sigma)=-\sigma+p_{-, \alpha}(G)$.

Proof.

(1) Given a manifold $M$, by forming the connected sum with copies of $\pm \mathbf{C} \mathbb{P}^{2}$ we can build a manifold with the same fundamental group but with $\sigma$ arbitrary. By mapping the extra $\pm \mathbf{C P}^{2}$ via the constant map to $B_{G}$, we can arrange that the class $\alpha \in H_{4}(G)$ is unchanged. Thus $q_{G}(\sigma)$ and $q_{G, \alpha}(\sigma)$ are defined for all $\sigma$.

(2) The parity statement follows from Proposition 3.1

(3) This is a restatement of Proposition 3.1.

(4) Since we can add $\pm \mathbf{C} \mathbb{P}^{2}$ to $M$ we see that $\left|q_{G}(\sigma+1)-q_{G}(\sigma)\right|=1$.

(5) This follows from the definition of $q_{G}$ as a minimum, the parity statement, and the fact that if $(\sigma, \chi) \in \mathcal{G}(G)$, then also $(\sigma, \chi+2) \in \mathcal{G}(G)$.

(6) The third assertion shows that the function $\sigma \mapsto q_{G}(\sigma)-\sigma$ is bounded below as $\sigma \rightarrow \infty$. The greatest lower bound is the Kotschick invariant $p(G)$. The fourth assertion shows that this integer-valued function is nonincreasing; in fact, it changes by 0 or -2 when $\sigma$ is replaced by $\sigma+1$. Hence it is eventually constant. The assertion for $q_{G}$ now follows. For $q_{G, \alpha}$ a separate (but duplicate) argument is needed for $\sigma \rightarrow-\infty$ since $q_{G, \alpha}$ may not be symmetric about the $\chi$-axis.

(7) This follows from the definition of $q(G)$.

(8) Note that $f_{G}(t) \neq-\infty$ if and only if $t \in[-1,1]$. To see this, recall Corollary 3.2 shows that if $(\sigma, \chi) \in \mathcal{G}(G)$, then so are $(\sigma-n, \chi+n)$ and $(\sigma+n, \chi+n)$ for all $n \in \mathbf{Z}$. For $t \in[-1,1]$, the half-plane $\chi+t \sigma \geq c$ contains $\mathcal{G}(G)$ whenever $c \leq f_{G}(t)$. Hence the convex hull of $\mathcal{G}(G)$ is the intersection of the half-planes $\chi+t \sigma \geq f_{G}(t), t \in[-1,1]$.

(9) Since $\mathcal{G}(G, \alpha) \subset \mathcal{G}(G), q_{G, \alpha}(\sigma) \geq q_{G}(\sigma)$.

(10) This is seen by reversing orientations.

\section{BASIC EXAMPLES}

4.1. The trivial group and free groups. We consider the trivial group, $\{e\}$ and observe that $\#_{a} \mathbf{C P}^{2} \#_{b}\left(-\mathbf{C P}^{2}\right)$ realizes any pair $(\sigma, \chi)$ satisfying Corollary 3.2 when $a=(\chi+\sigma-2) / 2$ and $b=(\chi-\sigma-2) / 2$. In Figure 2, we sketch the points of $\mathcal{G}(\{e\})$ contained in the region $[0,8] \times[0,8]$. We will explain later why the group $\mathbf{Z}^{3}$ has the same geography. Note that for both of these groups, $H_{4}(G)=0$, so that $\alpha=0$ is the only possibility. Thus

$$
q_{\{e\}}(\sigma)=|\sigma|+2=q_{\mathbf{Z}^{3}}(\sigma) .
$$

Consider next the free group on $n$ generators, $F_{n}$. Note that $H_{4}\left(F_{n}\right)=0$, $\beta_{1}\left(F_{n}\right)=n$, and $\beta_{2}\left(F_{n}\right)=0$. Theorem 3.4 implies that $q_{F_{n}}(\sigma) \geq|\sigma|-2 n+2$. The connected sum of $n$ copies of $S^{1} \times S^{3}$ with copies of $\pm \mathbf{C P} \mathbb{P}^{2}$ shows that this inequality is an equality:

$$
q_{F_{n}}(\sigma)=|\sigma|+2-2 n .
$$




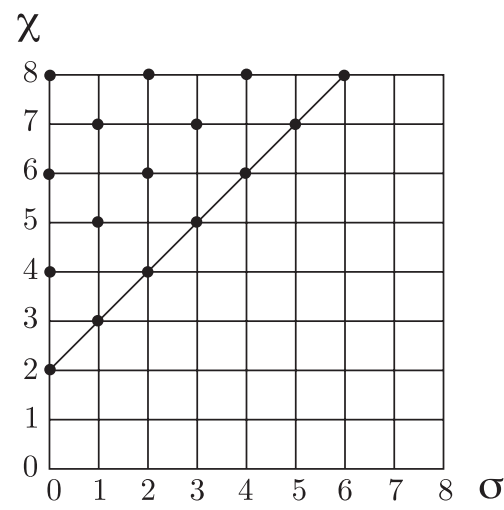

Figure 2. The geography of $\{e\}$ and $\mathbf{Z}^{3}$

4.2. Torsion classes; 2- and 3-manifold groups. The deficiency of a group $G$, $\operatorname{def}(G)$, is the supremum over all presentations of $G$ of $g-r$, where $g$ is the number of generators and $r$ is the number of relations.

In the case that $H_{4}(G)=0$ it was noted in [20] that in the second statement of Proposition 3.1. $\beta_{2}(G)$ can be replaced with $2 \beta_{2}(G): \chi(M) \geq 2 \beta_{2}(G)-2 \beta_{1}(G)+2$. A proof of a stronger result generalizing to $H_{4}(G ; F)$ with $F$ an arbitrary field appears in [22, Lemma 1.5]. See Section 4.3 below. Note that to control the signature it is necessary to work with $\mathbf{Z}$ (or $\mathbf{Q}$ ) coefficients. The idea of the proof is a straightforward generalization of the earlier proofs.

Proposition 4.1. Suppose $\alpha \in H_{4}(G)$ is a torsion class. Then

$$
q_{G, \alpha}(\sigma) \geq|\sigma|+2-2 \beta_{1}(G)+2 \beta_{2}(G) .
$$

In particular, if a group $G$ satisfies $H_{4}(G ; \mathbf{Q})=0$, then this inequality holds for any $\alpha \in H_{4}(G)$.

If moreover $\alpha=0$, then

$$
|\sigma|+2-2\left(\beta_{1}(G)-\beta_{2}(G)\right) \leq q_{G, 0}(\sigma) \leq|\sigma|+2-2 \operatorname{def}(G) .
$$

Proof. The cap product $\cap \alpha: H^{4}(G) \rightarrow \mathbf{Z}$ is zero when $\alpha$ is a torsion class. From Corollary 2.6 and Equation (2.4) one concludes that if $M \in \mathcal{M}(G,[\alpha])$, then the subspace $I(M)$ of $H^{2}(M) /$ torsion is isotropic.

Since the intersection form on $H^{2}(M) /$ torsion is unimodular, it follows that

$$
\operatorname{rank}\left(H^{2}(M)\right) \geq 2 \operatorname{rank}(I(M))+|\sigma(M)| .
$$

Proposition 2.4 implies that $I(M)$ is isomorphic to $H^{2}(G) /$ torsion, and so the rank of $I(M)$ equals $\beta_{2}(G)$. Thus $\beta_{2}(M) \geq 2 \beta_{2}(G)+|\sigma(M)|$, which implies the first inequality.

A presentation of a group $G$ with $g$ generators and $r$ relations determines a 2-handlebody $W^{4}$ with one 0 -handle, $g$ 1-handles, and $r$ 2-handles. Then the boundary $M=\partial\left(W^{4} \times[0,1]\right)$ also has fundamental group $G$ and $M \in \mathcal{M}(G,[0])$. Since $\chi(M)=2-2 \operatorname{def}(G)$ and $\sigma(M)=0, q_{G, 0}(0) \leq 2-2 \operatorname{def}(G)$. Taking connected sums with $\pm \mathbf{C P}^{2}$ gives the upper bound $q_{G, 0}(\sigma) \leq|\sigma|+2-2 \operatorname{def}(G)$.

As a quick application of Proposition 4.1, suppose $G$ is the fundamental group of a closed orientable surface $F$ of genus $g$. Then $H_{4}(G)=0$ and hence Proposition 4.1 
implies that when $g>0$ (so that $\beta_{2}(F)=\beta_{2}(G)$ ),

$|\sigma|+4-4 g=|\sigma|+2-2 \beta_{1}(G)+2 \beta_{2}(G) \leq q_{G}(\sigma) \leq|\sigma|+2-2 \operatorname{def}(G)=|\sigma|+4-4 g$.

This proves the following, extending [20, Proposition 5.4], which treats the cases of the invariants $p$ and $q$.

Theorem 4.2. If $G$ is the fundamental group of a closed orientable surface $F$ of genus greater than zero, then $H_{4}(G)=0$ and

$$
q_{G}(\sigma)=|\sigma|+2 \chi(F) .
$$

A more interesting class of examples is given by 3-manifold groups: we begin with the following well-known fact, which lets us apply Proposition 4.1

Proposition 4.3. If $G=\pi_{1}(N)$ for a compact 3-manifold $N$, then $H_{4}(G)=0$.

Proof. We present the proof only in the case of closed orientable 3-manifolds. Since $H_{4}\left(G_{1} * G_{2}\right)=H_{4}\left(G_{1}\right) \oplus H_{4}\left(G_{2}\right)$, we need consider only prime 3 -manifolds. Also, since $H_{4}(\mathbf{Z})=0$, we need not consider $S^{1} \times S^{2}$, and so consider only irreducible 3-manifolds.

If $G=\pi_{1}(M)$ is infinite and $M$ is an irreducible 3 -manifold, then by the sphere theorem, $M$ is an Eilenberg-Mac Lane space and $H_{4}(G)=0$. (See, for instance, [12.)

If $G=\pi_{1}(M)$ is finite and $M$ is irreducible, then the homology of $G$ is 4periodic, and $H_{4}(G)=\tilde{H}_{0}(G)=0$, where $\tilde{H}$ denotes the reduced homology. (See, for instance, [2].)

The following theorem completely characterizes the geography of closed oriented 3-manifold groups.

Theorem 4.4. Suppose $G$ is the fundamental group of a closed oriented 3-manifold, and assume that $G$ is a free product $G=F_{n} * H$, where $F_{n}$ is a free group on $n$ generators and $H$ is not a free product of any group with nontrivial free group. Then

$$
q_{G}(\sigma)=|\sigma|+2-2 n
$$

Proof. By Kneser's Conjecture 12, there is a a closed oriented 3-manifold $N$ with fundamental group $H$. Performing surgery on the circle $x \times S^{1} \subset N \times S^{1}$ yields a 4-manifold $M$ with $\pi_{1}(M)=H, \chi(M)=2$, and $\sigma(M)=0$. Taking the connected sum of $M$ with $n$ copies of $S^{1} \times S^{3}$ yields a 4-manifold with fundamental group $G$, Euler characteristic $2-2 n$, and signature zero. Thus $q_{G}(0) \leq 2-2 n$. Taking connected sums with $\pm \mathbf{C P}^{2}$ shows that

$$
q_{G}(\sigma) \leq|\sigma|+2-2 n .
$$

To prove the reverse inequality, we observe that the Eilenberg-Mac Lane space of a free product of two groups is the wedge of the Eilenberg-Mac Lane spaces of the factors. Using the Mayer-Vietoris sequence this implies

(4.1) $\beta_{1}(G)=\beta_{1}\left(F_{n}\right)+\beta_{1}(H)=n+\beta_{1}(H)$ and $\beta_{2}(G)=\beta_{2}\left(F_{n}\right)+\beta_{2}(H)=\beta_{2}(H)$.

The prime decomposition and sphere theorems for 3-manifolds imply that

$$
N=A_{1} \# \cdots \# A_{k} \# K_{1} \# \cdots \# K_{\ell} \#\left(S^{1} \times S^{2}\right)_{1} \# \cdots \#\left(S^{1} \times S^{2}\right)_{m},
$$


where the $A_{i}$ are aspherical and the $K_{i}$ have finite fundamental group (see for example [12]). Since we assumed that $H$ is not a free product, the prime decomposition of $N$ cannot have any $S^{1} \times S^{2}$ factors and so

$$
N=A_{1} \# \cdots \# A_{k} \# K_{1} \# \cdots \# K_{\ell} .
$$

Since $A_{i}$ is a closed, orientable, aspherical 3-manifold,

$$
\beta_{1}\left(\pi_{1}\left(A_{i}\right)\right)=\beta_{1}\left(A_{i}\right)=\beta_{2}\left(A_{i}\right)=\beta_{2}\left(\pi_{1}\left(A_{i}\right)\right) .
$$

Since $K_{i}$ has finite fundamental group,

$$
0=\beta_{1}\left(\pi_{1}\left(K_{i}\right)\right)=\beta_{1}\left(K_{i}\right)=\beta_{2}\left(K_{i}\right) \geq \beta_{2}\left(\pi_{1}\left(K_{i}\right)\right) \geq 0 .
$$

It follows that $\beta_{1}\left(\pi_{1}\left(K_{i}\right)\right)=0=\beta_{2}\left(\pi_{1}\left(K_{i}\right)\right)$. Using the Mayer-Vietoris sequence again, we compute

$$
\beta_{1}(H)=\sum_{j} \beta_{1}\left(\pi_{1}\left(A_{i}\right)\right)=\sum_{j} \beta_{2}\left(\pi_{1}\left(A_{i}\right)\right)=\beta_{2}(H) .
$$

Equations (4.1) and (4.2) and Proposition 4.1 imply that

$$
q_{G}(\sigma) \geq|\sigma|+2-2 n .
$$

Theorem 4.4 does not characterize closed oriented 3-manifold groups. One can construct examples as follows. Let $G$ be a finite group with periodic cohomology of period 4. Then $H_{4}(G)=0$. Corollary 4.4 of the article [9] by Hambleton and Kreck states that there exists a topological 4-dimensional rational homology sphere $M$ with fundamental group $G$. Since $\chi(M)=2$ and $\sigma(M)=0$, this example and Theorem 3.4 imply that $q_{G}(\sigma)=2+|\sigma|$. Milnor [25] listed groups with period 4 cohomology and showed that some of these groups, for example the symmetric group $G$ on three letters, are not the fundamental groups of 3-manifolds.

Some of these groups, though not fundamental groups of 3-manifolds, are the fundamental groups of 3-dimensional Poincaré complexes. For instance, Swan has shown in 29] that the symmetric group on three letters is the fundamental group of a 3-dimensional Poincaré complex. Thus these examples do not contradict the possibility that Theorem 4.4 characterizes 3-manifold groups in the Poincaré category. In addition, the manifolds constructed in [9] are topological 4-manifolds and it is not known whether smooth examples exist (see Problem 4.121 of [17]).

One can carry out calculations similar to those of Theorem 4.4 for the fundamental groups of not necessarily closed compact 3-manifolds; the statements become a bit more complicated. However, one interesting and simple example is the following.

Theorem 4.5. Let $K \subset S^{3}$ be a knot, and let $G=\pi_{1}\left(S^{3}-K\right)$. Then $H_{4}(G)=0$ and

$$
q_{G}(\sigma)=|\sigma| .
$$

Proof. A knot $K$ in $S^{3}$ has $H_{1}\left(S^{3}-K\right) \cong \mathbf{Z}$ and $H_{2}\left(S^{3}-K\right)=0$. Hence $\beta_{1}(G)-$ $\beta_{2}(G)=1$. The Wirtinger presentation of $G$ has deficiency 1 ; this easily implies $\operatorname{def}(G)=1$. The Sphere Theorem 12 implies that $S^{3}-K$ is an Eilenberg-Mac Lane space, and hence $H_{4}(G)=H_{4}\left(S^{3}-K\right)=0$. The last assertion of Proposition 4.1 then shows that $q_{G}(\sigma)=|\sigma|$. 
4.3. Multiple classes. The following is a well-known result, presented in print in [22, Lemma 1.5]; we state the theorem in terms of classes being multiples, $\alpha=p \tau$, rather than $\alpha=0 \in H_{4}(G ; \mathbf{Z} / p)$ to be consistent with our approach. The simple proof is included for the reader's convenience.

Theorem 4.6. Suppose $M \in \mathcal{M}(G,[\alpha])$ and that $\alpha \in H_{4}(G)$ is a multiple, say $\alpha=p \tau$ for some $\tau \in H_{4}(G)$ and prime $p$. Then

$$
\chi(M) \geq 2-2 \operatorname{dim}_{\mathbf{Z} / p}\left(H^{1}(G ; \mathbf{Z} / p)\right)+2 \operatorname{dim}_{\mathbf{Z} / p}\left(H^{2}(G ; \mathbf{Z} / p)\right) .
$$

In particular, if $H_{1}(G ; \mathbf{Z})$ has no $p$-torsion, then

$$
\chi(M) \geq 2-2 \beta_{1}(G)+2 \beta_{2}(G) .
$$

Proof. Let $f: M \rightarrow B_{G}$ denote the corresponding map. The homology group $H_{4}(M ; \mathbf{Z} / p)$ is isomorphic to $\mathbf{Z} / p$. The coefficient homomorphism $H_{4}(M ; \mathbf{Z}) \rightarrow$ $H_{4}(M ; \mathbf{Z} / p)$ induced by the surjection $\mathbf{Z} \rightarrow \mathbf{Z} / p$ takes the fundamental class $[M]$ to a generator, which we denote by $[M ; \mathbf{Z} / p]$. Poincaré duality implies that the intersection form with $\mathbf{Z} / p$ coefficients

$$
H^{2}(M ; \mathbf{Z} / p) \times H^{2}(M ; \mathbf{Z} / p) \rightarrow \mathbf{Z} / p,(x, y) \mapsto(x \cup y) \cap[M ; \mathbf{Z} / p]
$$

is nonsingular.

Since $\alpha=p \tau$, naturality of the coefficient homomorphism implies that $\alpha$ maps to zero under the morphism $f_{*}: H_{4}(G ; \mathbf{Z}) \rightarrow H_{4}(G ; \mathbf{Z} / p)$. Thus the $\mathbf{Z} / p$ intersection form vanishes on the subspace

$$
I=\operatorname{Image}\left(f^{*}: H^{2}(G ; \mathbf{Z} / p) \rightarrow H^{2}(M ; \mathbf{Z} / p)\right),
$$

since

$$
0=(x \cup y) \cap 0=(x \cup y) \cap f_{*}([M ; \mathbf{Z} / p])=\left(f^{*}(x) \cup f^{*}(y)\right) \cap[M ; \mathbf{Z} / p] .
$$

The subspace $I$ has the same $\mathbf{Z} / p$ dimension as $H^{2}(G ; \mathbf{Z} / p)$; in fact we have that $f^{*}: H^{2}(G ; \mathbf{Z} / p) \rightarrow H^{2}(M ; \mathbf{Z} / p)$ is injective since $B_{G}$ can be constructed by adding cells of dimension 3 and higher to $M$. Since the $\mathbf{Z} / p$ intersection form is nonsingular,

$$
\operatorname{dim}_{\mathbf{Z} / p} H^{2}(M ; \mathbf{Z} / p) \geq 2 \operatorname{dim}_{\mathbf{Z} / p} I .
$$

Computing the Euler characteristic of $M$ using $\mathbf{Z} / p$ coefficients and $\mathbf{Z} / p$ Poincaré duality yields

$$
\begin{aligned}
\chi(M) & =2-2 \operatorname{dim}_{\mathbf{Z} / p} H^{1}(M ; \mathbf{Z} / p)+\operatorname{dim}_{\mathbf{Z} / p} H^{2}(M ; \mathbf{Z} / p) \\
& \geq 2-2 \operatorname{dim}_{\mathbf{Z} / p} H^{1}(G ; \mathbf{Z} / p)+2 \operatorname{dim}_{\mathbf{Z} / p} H^{2}(G ; \mathbf{Z} / p) .
\end{aligned}
$$

The last statement follows from the universal coefficient theorem.

\section{Free ABELian Groups}

For the rest of this article we will focus on the geography of the free abelian groups. This class of groups provides a setting to investigate the ideas introduced above more deeply, and, as we shall see, will quickly lead us to difficult algebraic and 4-manifold problems. 
5.1. The homology and cohomology of $\mathbf{Z}^{n}$. We begin by reviewing the cohomology ring of $\mathbf{Z}^{n}$ and setting up notation and conventions.

A particular Eilenberg-Mac Lane space for $\mathbf{Z}^{n}$ is the $n$-torus, $B_{\mathbf{Z}^{n}}=\left(S^{1}\right)^{n}=T^{n}$. The cohomology ring $H^{*}\left(\mathbf{Z}^{n}\right)$ is the exterior algebra on $H^{1}\left(\mathbf{Z}^{n}\right)$. In particular, $H^{k}\left(\mathbf{Z}^{n}\right)$ is a free abelian group with rank the binomial coefficient, $C(n, k)$. When convenient, we fix a basis $\gamma_{1}, \cdots, \gamma_{n}$ of $\pi_{1}\left(T^{n}\right)=H_{1}\left(\mathbf{Z}^{n}\right)$. We denote the dual basis by $x_{1}, \cdots, x_{n} \in H^{1}\left(T^{n}\right)$. We drop the notation " $\cup$ " to indicate multiplication and so $H^{k}\left(\mathbf{Z}^{n}\right)$ has basis the $C(n, k)$ products $x_{i_{1}} x_{i_{2}} \cdots x_{i_{k}}, i_{1}<i_{2}<\cdots<i_{k}$.

The fact that $T^{n}$ is a closed orientable manifold gives us some additional algebraic structure, which we now describe. Note that the basis of $\pi_{1}\left(T^{n}\right)$ determines one of the two generators of $H_{n}\left(\mathbf{Z}^{n}\right) \cong \mathbf{Z}$ (that is, an orientation of $\left.T^{n}\right)$, and hence a fundamental class, which we denote by $[T] \in H_{n}\left(\mathbf{Z}^{n}\right)$. This then defines the duality isomorphism

$$
\cap[T]: H^{k}\left(\mathbf{Z}^{n}\right) \rightarrow H_{n-k}\left(\mathbf{Z}^{n}\right) .
$$

Given $\alpha \in H_{4}\left(\mathbf{Z}^{n}\right)$ denote by $\omega \in H^{n-4}\left(\mathbf{Z}^{n}\right)$ the Poincaré dual to $\alpha$, so

$$
\omega \cap[T]=\alpha \in H_{4}\left(\mathbf{Z}^{n}\right) .
$$

Given $x, y \in H^{2}\left(\mathbf{Z}^{n}\right)$,

$$
(x y) \cap \alpha=(x y \omega) \cap[T] .
$$

For our purposes, this formula is best recast in the following proposition, whose proof is just an application of the formula of Equation (2.4).

Proposition 5.1. Let $\alpha \in H_{4}\left(\mathbf{Z}^{n}\right)$ and choose $M \in \mathcal{M}\left(\mathbf{Z}^{n},[\alpha]\right)$. Let $\omega \in H^{n-4}\left(\mathbf{Z}^{n}\right)$ satisfy $\omega \cap[T]=\alpha$.

Then the restriction of the intersection form $H^{2}(M) \times H^{2}(M) \rightarrow \mathbf{Z}$ of $M$ to the subgroup $I(M) \subset H^{2}(M)$ is isometric to the pairing

$$
H^{2}\left(\mathbf{Z}^{n}\right) \times H^{2}\left(\mathbf{Z}^{n}\right) \rightarrow \mathbf{Z},(x, y) \mapsto x y \omega \cap[T] .
$$

The pairing of Proposition 5.1 does not depend on the orientation of $T^{n}$, since the intersection form of $M$ restricted to $I(M)$ is independent of the orientation of $T^{n}$. In any case it is useful to observe that changing the orientation of $T^{n}$ (for example, by changing the basis $\gamma_{1}, \cdots, \gamma_{n}$ of $\left.\pi_{1}(M)\right)$ changes the signs of both $\omega$ and $[T]$.

Notice the simplicity of this pairing: $H^{*}\left(\mathbf{Z}^{n}\right)=\Lambda^{*}\left(\mathbf{Z}^{n}\right)$, so an orientation of $T^{n}$ is just an identification $\Lambda^{n}\left(\mathbf{Z}^{n}\right) \cong \mathbf{Z}$, and the pairing of Proposition 5.1 in the usual notation of the exterior algebra is

$$
\Lambda^{2}\left(\mathbf{Z}^{n}\right) \times \Lambda^{2}\left(\mathbf{Z}^{n}\right) \rightarrow \mathbf{Z},(x, y) \mapsto x \wedge y \wedge \omega .
$$

Some basic consequences of this observation are assembled in the following theorem.

Theorem 5.2. Fix $\alpha \in H_{4}\left(\mathbf{Z}^{n}\right)$ and $M \in \mathcal{M}\left(\mathbf{Z}^{n},[\alpha]\right)$. Use the isomorphism $H^{1}\left(\mathbf{Z}^{n}\right) \rightarrow H^{1}(M)$ to identify the basis $\left\{x_{i}\right\}$ of $H^{1}\left(\mathbf{Z}^{n}\right)$ with a basis of $H^{1}(M)$.

Then the subspace $I(M) \subset H^{2}(M)$ is a free abelian summand of rank $C(n, 2)$. In the basis $\left\{x_{i} x_{j}\right\}_{i<j}$ of $I(M)$ one has $\left(x_{i} x_{j}\right)\left(x_{k} x_{\ell}\right)=0$ if an index is repeated in 
the set $\{i, j, k, \ell\}$. In particular:

(1) The restriction of the intersection form of $M$ to $I(M)$ is even.

(2) The intersection form of $M$ contains $(n-1)$-dimensional isotropic subspaces, for instance the subspace spanned by $\left\{x_{1} x_{2}, x_{1} x_{3}, \cdots, x_{1} x_{n}\right\}$. Hence $q_{\mathbf{Z}^{n}}(\sigma) \geq|\sigma|$.

(3) $\left(x_{i} x_{j}\right)\left(x_{k} x_{\ell}\right)=-\left(x_{i} x_{k}\right)\left(x_{j} x_{\ell}\right)$.

Proof. Let $f: M \rightarrow T^{n}$ induce the isomorphism on fundamental groups. Since $f^{*}: H^{2}\left(\mathbf{Z}^{n}\right) \rightarrow H^{2}(M)$ is injective by Proposition 2.4. $I(M) \subset H^{2}(M)$ is free abelian of rank $C(n, 2)$. To see that it is a summand, consider a map on the $n$-fold wedge

$$
j: S^{1} \vee \cdots \vee S^{1} \rightarrow M
$$

taking the $i$ th circle to a loop representing the generator $\gamma_{i} \in \pi_{1}(M)$. Since $\pi_{1}(M)=\mathbf{Z}^{n}$ is abelian, the map $j$ extends to the 2 -skeleton of the $n$-torus

$$
j:\left(T^{n}\right)^{(2)} \rightarrow M .
$$

The induced composite on cohomology

$$
H^{2}\left(\left(T^{n}\right)^{(2)}\right) \cong H^{2}\left(T^{n}\right) \stackrel{f^{*}}{\longrightarrow} H^{2}(M) \stackrel{j^{*}}{\longrightarrow} H^{2}\left(\left(T^{n}\right)^{(2)}\right)
$$

is clearly the identity and hence gives a splitting of $f^{*}$.

The rest of proof follows from Corollary 2.6 and basic properties of the exterior algebra, such as $x_{i} x_{j}=-x_{j} x_{i}$. In particular, since $\left(x_{i} x_{j}\right)\left(x_{i} x_{j}\right)=0$, the intersection form of $M$ restricted to $I(M)$ has zeros on the diagonal in this basis and hence is even. Notice that since the intersection form of $M$ has an $(n-1)$-dimensional isotropic subspace, $\beta_{2}(M) \geq|\sigma|+2(n-1)$, which implies that $\chi(M) \geq|\sigma|$.

Corollary 5.3. Suppose that $\alpha \in H_{4}\left(\mathbf{Z}^{n}\right)$ and that there exists an $M \in \mathcal{M}\left(\mathbf{Z}^{n},[\alpha]\right)$ so that $H^{2}\left(\mathbf{Z}^{n}\right) \rightarrow H^{2}(M)$ is surjective. Then $n \neq 2,3$, and the intersection form of $M$ is equivalent to $k E_{8} \oplus \ell H$ for some $k \in \mathbf{Z}$ and $\ell \geq n-1$. In particular $C(n, 2)=8|k|+2 \ell$ and therefore is even. If $M$ is smooth, then in addition $k$ is even.

Proof. If $f^{*}: H^{2}\left(T^{n}\right) \rightarrow H^{2}(M)$ is surjective, then by Theorem 5.2 it is an isomorphism and the intersection form of $M$ is even. Since it contains an $(n-1)$ dimensional isotropic subspace, $C(n, 2) \geq 2(n-1)$, and hence $n \neq 2,3$. Furthermore the intersection form is indefinite, so the classification of even, unimodular integer bilinear forms shows that the intersection form of $M$ is equivalent to $k E_{8} \oplus \ell H$ for some $k \in \mathbf{Z}$ and $\ell \geq n-1$.

If $M$ is smooth, then since $H_{1}(M)$ has no 2-torsion, it follows that $M$ is spin, so that by Rohlin's theorem the signature of $M$ is divisible by 16 . Hence $k$ is even.

Theorem 5.2 and Corollary 5.3 together imply that

$$
q\left(\mathbf{Z}^{n}\right) \geq 2-2 n+C(n, 2)+\epsilon_{n},
$$

where $\epsilon_{n}$ equals zero if $C(n, 2)$ is even and equals 1 if $C(n, 2)$ is odd. The main result of [19] is that this lower bound is achieved for all $n$, except that $q\left(\mathbf{Z}^{3}\right)=2$, rather than 0 , and $q\left(\mathbf{Z}^{5}\right)=6$, rather than 2. Moreover, the examples constructed in 19. which realize this lower bound all have signature zero since they are obtained by 
surgeries on connected sums of products of orientable surfaces. Thus we conclude that for $n \neq 3,5$,

$$
q_{\mathbf{Z}^{n}}(\sigma) \geq q_{\mathbf{Z}^{n}}(0)=2-2 n+C(n, 2)+\epsilon_{n} .
$$

Example. Suppose $\omega \in H^{4}\left(\mathbf{Z}^{8}\right)$ so that the induced pairing (5.1) is unimodular. Notice that such an $\omega$ exists by the results of [19] since $C(8,2)=28$ is even. The classification theorem for unimodular integer forms [26] shows that this pairing is equivalent (after perhaps changing orientation) to either $14 H$ or $E_{8} \oplus 10 H$.

Now if $M \in \mathcal{M}\left(\mathbf{Z}^{8}, \omega \cap[T]\right)$ satisfies $\beta_{2}(M)=28$, then $I(M)=H^{2}(M)$ and so the intersection form of $M$ is equivalent to (5.1). The example constructed in [19] has signature zero, hence has intersection form $14 H$, but Corollary 5.3 allows the possibility that for some $\omega$ the signature equals 8 .

\subsection{Calculations.}

5.2.1. $\mathbf{n}=\mathbf{0 , 1}, \mathbf{2}, \mathbf{3}$. Since $\mathbf{Z}^{0}, \mathbf{Z}^{1}$ and $\mathbf{Z}^{3}$ are closed 3 -manifold groups (the corresponding 3-manifolds are $S^{3}, S^{1} \times S^{2}$, and $T^{3}$ ) and $\mathbf{Z}^{2}$ is the fundamental group of a torus, the geography for these groups is given by Theorems 4.4 and 4.2 .

$$
q_{\mathbf{Z}^{n}}(\sigma)= \begin{cases}|\sigma|+2 & \text { if } n=0,3, \\ |\sigma| & \text { if } n=1,2 .\end{cases}
$$

The geography of $\mathbf{Z}^{0}$ and $\mathbf{Z}^{3}$ has already been illustrated in Figure 2, and the geography of $\mathbf{Z}^{1}, \mathbf{Z}^{2}$, and (as we will show next), $\mathbf{Z}^{4}$ is illustrated in Figure 3 .

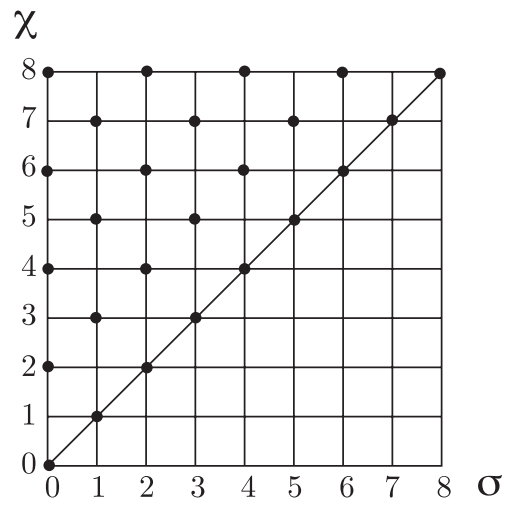

Figure 3. The geography of $\mathbf{Z}, \mathbf{Z}^{2}$, and $\mathbf{Z}^{4}$

5.2.2. $\mathbf{n}=4$. First note that $H_{4}\left(\mathbf{Z}^{4}\right)=\mathbf{Z}$. Moreover, since the 4 -torus admits an orientation reversing homeomorphism, $q_{\mathbf{Z}^{4}, \alpha}(-\sigma)=q_{\mathbf{Z}^{4},-\alpha}(\sigma)=q_{\mathbf{Z}^{4}, \alpha}(\sigma)$ for any $\alpha \in H_{4}\left(\mathbf{Z}^{4}\right)$.

Since $\beta_{2}\left(\mathbf{Z}^{4}\right)=C(4,2)=6$, Theorem 5.2 implies that for any $\alpha \in H_{4}\left(\mathbf{Z}^{4}\right)$ and any $M \in \mathcal{M}\left(\mathbf{Z}^{4},[\alpha]\right), \beta_{2}(M) \geq 6$. Theorem 5.2 shows that $q_{\mathbf{Z}^{4}, \alpha}(\sigma) \geq|\sigma|$. Taking the identity map of the 4 -torus and connected sums with $\pm \mathbf{C} \mathbb{P}^{2}$ shows that

$$
q_{\mathbf{Z}^{4}}(\sigma)=|\sigma|
$$

and that if $[T] \in H_{4}\left(\mathbf{Z}^{4}\right)$ is a generator, then

$$
q_{\mathbf{Z}^{4},[T]}(\sigma)=|\sigma| .
$$


If $\alpha=k[T]$ for $|k|>1$ or $k=0$, then Theorem 4.6 implies that $\chi(M) \geq$ $2-8+12=6$ and so $q_{\mathbf{Z}^{4}, \alpha}(\sigma) \geq \max \{6,|\sigma|\}$.

A signature zero example with $\chi=6$ representing $\alpha=k[T],|k| \neq 1$, can be constructed as follows. Let $M^{\prime}:=T^{4} \#\left(S^{1} \times S^{3}\right)$, with $\pi_{1}\left(T^{4}\right)$ generated by $a_{1}, a_{2}, a_{3}, a_{4}$ and $\pi_{1}\left(S^{1} \times S^{3}\right)$ generated by $b$. Let $f^{\prime}: M^{\prime} \rightarrow T^{4}$ be a map inducing the map $a_{1} \mapsto \gamma_{1}, a_{2} \mapsto \gamma_{2}, a_{3} \mapsto \gamma_{3}, a_{4} \mapsto \gamma_{4}^{k}, b \mapsto \gamma_{4}$ on fundamental groups. Then $f_{*}^{\prime}\left(\left[M^{\prime}\right]\right)=k[T]$ and $f^{\prime}$ induces an epimorphism on fundamental groups. Note that $\chi\left(M^{\prime}\right)=-2$ and $\sigma\left(M^{\prime}\right)=0$. Now perform 4 surgeries along circles in $M^{\prime}$ : the first to introduce the relation $b^{k}=a_{4}$ and the other three to introduce the commutator relations $\left[a_{1}, b\right],\left[a_{2}, b\right]$ and $\left[a_{3}, b\right]$. Each surgery increases the Euler characteristic by 2 and leaves the signature invariant. The map $f^{\prime}$ clearly extends over the trace of the surgery. Thus the resulting 4-manifold $M$ has a map $f: M \rightarrow T^{4}$ inducing an isomorphism on fundamental groups, and representing $k[T]$. Moreover, $\chi(M)=6$ and $\sigma(M)=0$.

Thus $q_{\mathbf{Z}^{4}, k[T]}(0)=6$ for $|k| \neq 1$. Taking connected sums with $\pm \mathbf{C P}^{2}$ shows that

$$
\max \{6,|\sigma|\} \leq q_{\mathbf{Z}^{4}, k[T]}(\sigma) \leq|\sigma|+6 .
$$

When $k=0$, i.e. $M \in \mathcal{M}\left(\mathbf{Z}^{4}, 0\right)$, then $I(M)$ is a 6 -dimensional isotropic subspace. Hence

$$
q_{\mathbf{Z}^{4}, 0}(\sigma)=|\sigma|+6 .
$$

We summarize these calculations in the following theorem.

Theorem 5.4. Given $\alpha \in H_{4}\left(\mathbf{Z}^{4}\right)$, define the nonnegative integer $k \geq 0$ so that $\pm \alpha=k[T]$. Then the following hold.

(1) $q_{\mathbf{Z}^{4}}(\sigma)=|\sigma|$, and so $q\left(\mathbf{Z}^{4}\right)=0, p\left(\mathbf{Z}^{4}\right)=0$.

(2) If $k=0, q_{\mathbf{Z}^{4}, \alpha}(\sigma)=|\sigma|+6$.

(3) If $k=1, q_{\mathbf{Z}^{4}, \alpha}(\sigma)=|\sigma|$.

(4) If $k>1, \max \{6,|\sigma|\} \leq q_{\mathbf{Z}^{4}, \alpha}(\sigma) \leq|\sigma|+6$.

Question. Is $q_{\mathbf{Z}^{4}, k[T]}(\sigma)=|\sigma|+6$ for $|k|>1$ ? From parity considerations we know that $q_{\mathbf{Z}^{4}, k[T]}(1)=7$ when $|k| \neq 1$. We do not know whether $q_{\mathbf{Z}^{4}, k[T]}(2)=6$ or 8 .

The unknown values of $q_{\mathbf{Z}^{4}, k[T]}$ for $|k|>1$ are marked with circles in Figure 4 ,

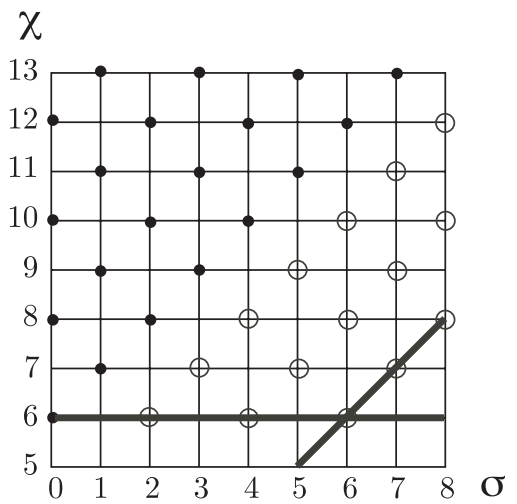

Figure 4. The unknown points in $\mathcal{G}\left(\mathbf{Z}^{4}, k[T]\right),|k| \geq 2$ 
5.2.3. $\mathbf{n}=\mathbf{5}$. Note that $H^{2}\left(\mathbf{Z}^{5}\right)$ has rank 10 and $H_{4}\left(\mathbf{Z}^{5}\right)$ has rank 5. We first claim that given any $\alpha \in H_{4}\left(\mathbf{Z}^{5}\right)$, there is an automorphism $\Psi \in \operatorname{Aut}\left(\mathbf{Z}^{5}\right)$ so that $\Psi_{*}(\alpha)=\left(k x_{1}\right) \cap[T]$ for some $k \in \mathbf{Z}$.

The following lemma helps to keep track of how automorphisms of $\pi_{1}\left(T^{n}\right)=$ $H_{1}\left(T^{n}\right)$ act on the cohomology $H^{*}\left(T^{n}\right)$, thinking of the cohomology as the exterior algebra.

Lemma 5.5. Let $A: \pi_{1}\left(T^{n}\right) \rightarrow \pi_{1}\left(T^{n}\right)$ be an automorphism, given in the basis $\gamma_{1} \cdots, \gamma_{n}$ as an $n \times n$ matrix $A \in G L\left(\mathbf{Z}^{n}\right)$. Let $x_{1}, \cdots, x_{n} \in H^{1}\left(\mathbf{Z}^{n}\right)=$ $\operatorname{Hom}\left(\pi_{1}\left(T^{n}\right), \mathbf{Z}\right)$ denote the dual basis. Then the induced map $A^{*}: H^{1}\left(\mathbf{Z}^{n}\right) \rightarrow$ $H^{1}\left(\mathbf{Z}^{n}\right)$ is given by the transpose $A^{T}$ in the basis $\left\{x_{i}\right\}$.

Moreover, if $\omega \in H^{n-4}\left(\mathbf{Z}^{n}\right), \alpha \in H_{4}\left(\mathbf{Z}^{n}\right)$ satisfy $\omega \cap[T]=\alpha$, then $A_{*}(\alpha)=$ $\operatorname{det}(A)\left(\left(A^{*}\right)^{-1}(\omega)\right) \cap[T]$.

Proof. The fact that $A^{*}=A^{T}$ is well known and easy. The other formula is a consequence of the naturality of cap products, expressed by the identity $f_{*}\left(f^{*}(x) \cap\right.$ $y)=x \cap f_{*}(y)$, valid for any continuous map $f$. In more detail, taking $f: T^{n} \rightarrow T^{n}$ to be a map inducing the automorphism $A: \pi_{1}\left(T^{n}\right) \rightarrow \pi_{1}\left(T^{n}\right)$, the induced map $A_{*}: H_{n}\left(\mathbf{Z}^{n}\right) \rightarrow H_{n}\left(\mathbf{Z}^{n}\right)$ is multiplication by $\operatorname{det}(A)$. One computes

$$
\begin{aligned}
A_{*}(\alpha) & =A_{*}(\omega \cap[T])=A_{*}\left(A^{*}\left(A^{*}\right)^{-1}(\omega) \cap[T]\right) \\
& =\left(A^{*}\right)^{-1}(\omega) \cap A_{*}([T])=\left(A^{*}\right)^{-1}(\omega) \cap \operatorname{det}(A)[T] .
\end{aligned}
$$

We use Lemma [5.5 as follows. Given $\alpha \in H_{4}\left(\mathbf{Z}^{5}\right)$, let $\omega \in H^{1}\left(\mathbf{Z}^{5}\right)$ satisfy $\omega \cap[T]=\alpha$. Since $\omega \in H^{1}\left(\mathbf{Z}^{5}\right)$, we can write $\omega=k\left(b_{1} x_{1}+\cdots+b_{5} x_{5}\right)$ where $k \in \mathbf{Z}$ and $b_{1} x_{1}+\cdots+b_{5} x_{5}$ is primitive (that is, not divisible). Thus there is some matrix $B \in G L\left(\mathbf{Z}^{5}\right)$ which sends $\omega$ to $k x_{1}$. Clearly we can assume $\operatorname{det}(B)=1$ and $k \geq 0$. Lemma $\left[5.5\right.$ implies that the matrix $A=\left(B^{T}\right)^{-1}$ determines an automorphism $\Psi: \mathbf{Z}^{5} \rightarrow \mathbf{Z}^{5}$ satisfying $\Psi_{*}(\alpha)=k x_{1} \cap[T]$.

Since $\mathcal{G}\left(\mathbf{Z}^{5}, \alpha\right)=\mathcal{G}\left(\mathbf{Z}^{5}, \Psi_{*}(\alpha)\right)$, we see that $\mathcal{G}\left(\mathbf{Z}^{5}, \alpha\right)=\mathcal{G}\left(\mathbf{Z}^{5}, k x_{1} \cap[T]\right)$, where $k \geq 0$ is the unique integer so that $\alpha=k \tau$ for $\tau$ a primitive class.

If $\alpha=0$, then $I(M)$ is a 10 -dimensional isotropic subspace for any $M \in$ $\mathcal{M}\left(\mathbf{Z}^{n},[0]\right)$. For such an $M,|\sigma| \leq \beta_{2}(M)-20$, and so

$$
q_{\mathbf{Z}^{5}, 0}(\sigma)=2-10+\beta_{2}(M) \geq|\sigma|+12 .
$$

If $\alpha \neq 0$, then since $x y \cap \alpha=x y \omega \cap\left[T^{5}\right]=k x y x_{1} \cap[T]$, the intersection form vanishes on the subspace of $I(M)$ generated by

$$
\left\{x_{1} x_{2}, x_{1} x_{3}, x_{1} x_{4}, x_{1} x_{5}, x_{2} x_{3}, x_{2} x_{4}, x_{2} x_{5}\right\},
$$

which is of rank 7. Thus, for any $M \in \mathcal{M}\left(\mathbf{Z}^{5},[\alpha]\right)$ with $\alpha \neq 0, \chi(M)=2-10+$ $\beta_{2}(M) \geq-8+|\sigma(M)|+14=|\sigma(M)|+6$. Hence for $\alpha \neq 0$,

$$
q_{\mathbf{Z}^{5}, \alpha}(\sigma) \geq|\sigma|+6 \text {. }
$$

To construct an upper bound, take $M^{\prime}=T^{4} \#\left(T^{2} \times S^{2}\right)$ with $\pi_{1}\left(T^{4}\right)$ generated by $a_{1}, a_{2}, a_{3}, a_{4}$ and $\pi_{1}\left(T^{2} \times S^{2}\right)$ generated by $b_{1}, b_{2}$. Thus $\chi\left(M^{\prime}\right)=-2$ and $\sigma\left(M^{\prime}\right)=0$. Select a map $M^{\prime} \rightarrow T^{5}$ inducing the homomorphism $a_{1} \mapsto \gamma_{2}, a_{2} \mapsto \gamma_{3}, a_{3} \mapsto$ $\gamma_{4}, a_{4} \mapsto \gamma_{5}^{k}, b_{1} \mapsto \gamma_{1}, b_{2} \mapsto \gamma_{5}$ on fundamental groups. Then $\alpha=k x_{1} \cap[T]$.

If $\alpha=0$ (in other words, $k=0$ ), perform 7 surgeries on $M^{\prime}$ : one to kill $a_{4}$ and six to kill the commutators of $a_{1}, a_{2}, a_{3}$ with $b_{1}, b_{2}$. Each surgery increases $\chi$ by 2 . Clearly the map from the resulting manifold $M$ to $T^{5}$ induces an isomorphism and 
represents 0 in $H_{4}\left(\mathbf{Z}^{5}\right)$. Since $\chi(M)=12$ and $\sigma(M)=0$, taking connected sums with $\pm \mathbf{C P}^{2}$ provides examples showing $q_{\mathbf{Z}^{5}, 0}(\sigma) \leq|\sigma|+12$, and so

$$
q_{\mathbf{Z}^{5}, 0}(\sigma)=|\sigma|+12 .
$$

If $\alpha=x_{1} \cap[T]$, i.e. $k=1$, perform 4 surgeries on $M^{\prime}$ : one to kill $b_{2} a_{4}^{-1}$, and three to kill the commutators $\left[a_{1}, b_{1}\right],\left[a_{2}, b_{1}\right]$ and $\left[a_{3}, b_{1}\right]$. The map from the resulting manifold $M$ to $T^{5}$ induces an isomorphism and represents $\alpha$ in $H_{4}\left(\mathbf{Z}^{5}\right)$. Since $\chi(M)=6$ and $\sigma(M)=0$, taking connected sums with $\pm \mathbf{C P}^{2}$ provides examples showing $q_{\mathbf{Z}^{5}, 0}(\sigma) \leq|\sigma|+6$. Thus for $k=1$ :

$$
q_{\mathbf{Z}^{5}, x_{1} \cap[T]}(\sigma)=|\sigma|+6 .
$$

If $k>1$, perform 7 surgeries on $M^{\prime}$ : one to kill $b_{1}^{k} a_{4}^{-1}$, and six to kill the commutators $\left[a_{1}, b_{1}\right],\left[a_{2}, b_{1}\right],\left[a_{3}, b_{1}\right],\left[a_{1}, b_{2}\right],\left[a_{2}, b_{2}\right]$, and $\left[a_{3}, b_{2}\right]$. The map from the resulting manifold $M$ to $T^{5}$ induces an isomorphism and represents $\alpha$ in $H_{4}\left(\mathbf{Z}^{5}\right)$. Since $\chi(M)=12$ and $\sigma(M)=0$, taking connected sums with $\pm \mathbf{C P}^{2}$ provides examples showing $q_{\mathbf{Z}^{5}, k x_{1} \cap[T]}(\sigma) \leq|\sigma|+12$. On the other hand, Theorem 4.6] shows that for such $\alpha, \chi(M) \geq 12$.

Thus for $k>1$ :

$$
\max \{12,|\sigma|+6\} \leq q_{\mathbf{Z}^{5}, k x_{1} \cap[T]}(\sigma) \leq|\sigma|+12 .
$$

Taking the minimum over all homology classes $\alpha$ we see that

$$
q_{\mathbf{Z}^{5}}(\sigma)=|\sigma|+6 .
$$

In summary:

Theorem 5.6. Given $\alpha \in H_{4}\left(\mathbf{Z}^{5}\right)$, there exists a unique nonnegative integer $k$ and $A \in \operatorname{Aut}\left(\mathbf{Z}^{5}\right)$ so that $A_{*}(\alpha)=\left(k x_{1}\right) \cap[T]$. Then the following hold.

(1) $q_{\mathbf{Z}^{5}}(\sigma)=|\sigma|+6$, and so $q\left(\mathbf{Z}^{5}\right)=6, p\left(\mathbf{Z}^{5}\right)=6$.

(2) If $k=0, q_{\mathbf{Z}^{5}, 0}(\sigma)=|\sigma|+12$.

(3) If $k=1, q_{\mathbf{Z}^{5}, x_{1} \cap[T]}(\sigma)=|\sigma|+6$.

(4) If $k>1, \max \{12,|\sigma|+6\} \leq q_{\mathbf{Z}^{5}, k x_{1} \cap[T]}(\sigma) \leq|\sigma|+12$.

Question. If $k>1$, is $q_{\mathbf{Z}^{5}, k x_{1} \cap[T]}(\sigma)=|\sigma|+12$ ?

The geography of $\mathbf{Z}^{5}$ is illustrated in Figure 5 . (Note the change in the vertical scale in the figure.)

5.2.4. $\mathbf{n}=6$ 6. For $\mathbf{Z}^{6}$, the situation becomes more delicate and interesting; for instance, $q_{\mathbf{Z}^{6}}(\sigma)$ is not linear for $\sigma \geq 0$. If $\pi_{1}(M)=\mathbf{Z}^{6}$, then $I(M)$ has a 7 dimensional isotropic subspace and it follows that $\max \{6,|\sigma|+4\} \leq q_{\mathbf{Z}^{6}}(\sigma)$ (see the proof of Theorem 5.9 and Equation (5.4) $)$.

It is convenient at this point to recall the examples of symmetric products, [1, 24.

Proposition 5.7. Let $F_{k}$ denote the oriented compact surface of genus $k$. Let $S_{2 k}=\operatorname{Sym}^{2}\left(F_{k}\right)$. Then $S_{2 k}$ is a smooth 4-manifold with $\pi_{1}\left(S_{2 k}\right)=\mathbf{Z}^{2 k}, \chi\left(S_{2 k}\right)=$ $2 k^{2}-5 k+3$ and $\sigma\left(S_{2 k}\right)=1-k$.

A 4 -manifold $M$ is constructed in [19] with $\pi_{1}(M)=\mathbf{Z}^{6}, \chi(M)=6$ and $\sigma(M)=$ 0 (see also the proof of Theorem 5.9 below). It follows that $q_{\mathbf{Z}^{6}}(0)=6$. Since 


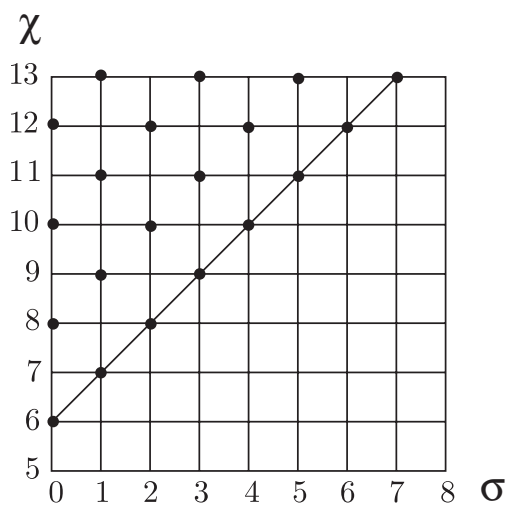

Figure 5. The geography of $\mathbf{Z}^{5}$

$q_{\mathbf{Z}^{6}}(1) \geq 6$, it follows that $q_{\mathbf{Z}^{6}}(1)=7$. The 4 -manifold $S_{6}$ has fundamental group $\mathbf{Z}^{6}, \chi\left(S_{6}\right)=6$, and $\sigma\left(S_{6}\right)=-2$. Thus, $q_{\mathbf{Z}^{6}}(2)=q_{\mathbf{Z}^{6}}(-2)=6$. It follows that

$$
q_{\mathbf{Z}^{6}}(\sigma)= \begin{cases}6 & \sigma=0 \\ 7 & |\sigma|=1 \\ |\sigma|+4 & |\sigma| \geq 2\end{cases}
$$

as illustrated in Figure 6

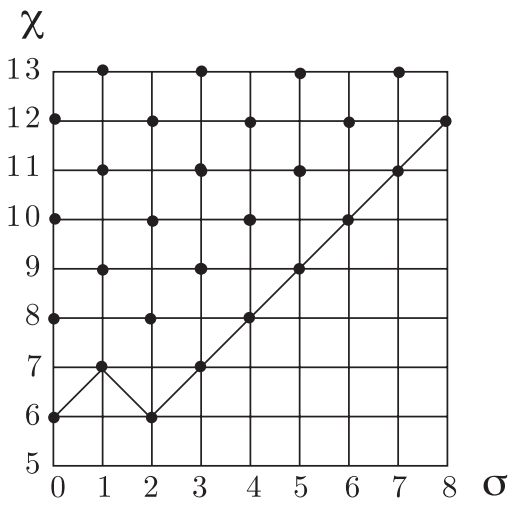

Figure 6 . The geography of $\mathbf{Z}^{6}$

To compute $q_{\mathbf{Z}^{6}, \alpha}$, we find a canonical form for the homology classes $\alpha \in H^{2}\left(\mathbf{Z}^{6}\right)$. In the following theorem, we fix a basis $\gamma_{1}, \ldots, \gamma_{6} \in H_{1}\left(\mathbf{Z}^{6}\right)$ and let $x_{1}, \cdots, x_{6} \in$ $H^{1}\left(\mathbf{Z}^{6}\right)$ denote the dual basis. These determine an orientation class $[T] \in H_{6}\left(\mathbf{Z}^{6}\right)$.

For a triple of integers $a, b, c$, the notation $a|b| c$ means that there are integers $k, \ell$ so that $b=a k$ and $c=b \ell$.

Theorem 5.8. Given any $\alpha \in H_{4}\left(\mathbf{Z}^{6}\right)$, there exists an automorphism $A \in \operatorname{Aut}\left(\mathbf{Z}^{6}\right)$ and $a$ unique set of integers $a, b, c \in \mathbf{Z}$ with $a|b| c, a, b \geq 0$, so that

$$
A_{*}(\alpha)=\left(a x_{1} x_{2}+b x_{3} x_{4}+c x_{5} x_{6}\right) \cap[T] .
$$


Proof. Let $B \in S L_{6}(\mathbf{Z})$. In the basis $\left\{x_{i}\right\}, B$ defines automorphisms $B^{*}: H^{1}\left(\mathbf{Z}^{6}\right) \rightarrow$ $H^{1}\left(\mathbf{Z}^{6}\right)$ and $B^{*}: H^{2}\left(\mathbf{Z}^{6}\right) \rightarrow H^{2}\left(\mathbf{Z}^{6}\right)$ by $B^{*}\left(x_{i}\right)=\sum_{j} B_{i j} x_{j}$ and $B^{*}\left(x_{i} x_{j}\right)=$ $B^{*}\left(x_{i}\right) B^{*}\left(x_{j}\right)$. Let $\omega \in H^{2}\left(\mathbf{Z}^{6}\right)$ satisfy $\omega \cap[T]=\alpha$. Lemma 5.5 implies that if we set $A=\left(B^{T}\right)^{-1}$, the automorphism $A: \mathbf{Z}^{6} \rightarrow \mathbf{Z}^{6}$ given by $A\left(\gamma_{i}\right)=\sum_{j} A_{i, j} \gamma_{j}$ satisfies $A_{*}(\alpha)=B^{*}(\omega) \cap[T]$.

We first prove that for any $\omega \in H^{2}\left(\mathbf{Z}^{6}\right)$, there is a matrix $B \in S L_{6}(\mathbf{Z})$ so that $B^{*}(\omega)$ is of the form $a x_{1} x_{2}+b x_{3} x_{4}+c x_{5} x_{6}$ for some $a, b$, and $c$.

Any $\omega \in H^{2}\left(\mathbf{Z}^{6}\right)$ can be written as $\sum \alpha_{i, j} x_{i} x_{j}, i<j$. Collecting terms, we can express $\omega$ as

$$
\omega=a_{1} x_{1}\left(\alpha_{2} x_{2}+\cdots+\alpha_{6} x_{6}\right)+\omega_{2},
$$

where the $\alpha_{i}$ have gcd $=1, a_{1} \geq 0$, and $\omega_{2}$ can be expressed in terms of $x_{i} x_{j}$ with $2 \leq i<j$. (If $x_{1}$ appears in $\omega$, then $a_{1}>0$ and the $\alpha_{i}$ are uniquely determined; otherwise, we have the decomposition $\omega=0 x_{1}\left(x_{2}\right)+\omega_{2}$.)

Within $\left\langle x_{2}, \ldots, x_{6}\right\rangle \cong \mathbf{Z}^{5}$ there is a basis with its first element $\alpha_{2} x_{2}+\cdots+\alpha_{6} x_{6}$. Renaming those basis elements $x_{2}, \ldots, x_{6}$ gives

$$
\omega=a_{1} x_{1} x_{2}+\omega_{2},
$$

where $\omega_{2}$ continues to be expressed in terms of $x_{i} x_{j}, 2 \leq i<j$.

Repeating this process, we can change the basis so that

$$
\omega=a_{1} x_{1} x_{2}+a_{2} x_{2} x_{3}+a_{3} x_{3} x_{4}+a_{4} x_{4} x_{5}+a_{5} x_{5} x_{6}
$$

and by changing signs of the appropriate $x_{i}$ we can arrange that $a_{i} \geq 0$ for all $i$.

Now we want to see that by a further change of basis it can be assumed that either $a_{1}=0$ or $a_{2}=0$. We will demonstrate this by repeatedly changing basis so that $\min \left\{a_{1}, a_{2}\right\}$ is reduced.

Suppose now that $a_{1} \leq a_{2}$. Make the change of basis $x_{1} \mapsto x_{1}+x_{3}$. Then we have:

$$
\omega=a_{1} x_{1} x_{2}+\left(a_{2}-a_{1}\right) x_{2} x_{3}+a_{3} x_{3} x_{4}+a_{4} x_{4} x_{5}+a_{5} x_{5} x_{6} .
$$

The coefficient on $x_{2} x_{3}$ became smaller, and this can be repeated until the coefficient on $x_{2} x_{3}$ is smaller than $a_{1}$.

On the other hand, if $a_{2}<a_{1}$, make the change of basis $x_{2} \mapsto x_{1}+x_{2}$. Then we have

$$
\omega=x_{1}\left(a_{1} x_{2}+a_{2} x_{3}\right)+a_{2} x_{2} x_{3}+a_{3} x_{3} x_{4}+a_{4} x_{4} x_{5}+a_{5} x_{5} x_{6} .
$$

Now, factor out $a_{1}^{\prime}=\operatorname{gcd}\left(a_{1}, a_{2}\right)$ (and note that $\left.a_{1}^{\prime} \leq a_{2}\right)$ to write

$$
\omega=a_{1}^{\prime} x_{1}\left(\alpha_{1} x_{2}+\alpha_{2} x_{3}\right)+a_{2} x_{2} x_{3}+a_{3} x_{3} x_{4}+a_{4} x_{4} x_{5}+a_{5} x_{5} x_{6},
$$

with $\alpha_{1}$ and $\alpha_{2}$ relatively prime. As earlier, a change of basis on $\left\langle x_{2}, \ldots, x_{6}\right\rangle$ can be made so that $\alpha_{2} x_{2}+\alpha_{3} x_{3}$ is replaced by $x_{2}$ and with respect to this new basis, we have

$$
\omega=a_{1}^{\prime} x_{1} x_{2}+a_{2}^{\prime} x_{2} x_{3}+a_{3}^{\prime} x_{3} x_{4}+a_{4}^{\prime} x_{4} x_{5}+a_{5}^{\prime} x_{5} x_{6} .
$$

Notice that $a_{2}^{\prime}$ may be greater than $a_{2}$, but we do have that $a_{1}^{\prime} \leq a_{2}<a_{1}$. If $a_{1}^{\prime}<a_{2}$ we are done. Otherwise, do the earlier change of basis to lower $a_{2}^{\prime}$ by a multiple of $a_{1}^{\prime}$ so that $a_{2}^{\prime}<a_{1}^{\prime}$ and thus $a_{2}^{\prime}<a_{1}^{\prime} \leq a_{2}<a_{1}$. Hence the smaller of the two decreased. Continuing in this manner we obtain either $a_{1}=0$ or $a_{2}=0$.

With this, it follows from induction that $\omega \in H^{2}\left(\mathbf{Z}^{6}\right)$ can be put in the form $\omega=a x_{1} x_{2}+b x_{3} x_{4}+c x_{5} x_{6}$ for some $a, b$, and $c$, with respect to some basis.

We next want to achieve the desired divisibility of the coefficients. Consider $c\left(a x_{1} x_{2}+b x_{3} x_{4}\right) \in H^{2}\left(\mathbf{Z}^{4}\right)$ with $a, b, c \in \mathbf{Z}$ and $a$ and $b$ relatively prime. Choose 
integers $p, q$ with $a p+b q=1$. The determinant 1 automorphism defined by the substitutions

$$
x_{1}=x_{1}^{\prime}-b q x_{3}^{\prime}, \quad x_{2}=p x_{2}^{\prime}-b x_{4}^{\prime}, \quad x_{3}=x_{1}^{\prime}+a p x_{3}^{\prime}, \quad x_{4}=q x_{2}^{\prime}+a x_{4}^{\prime}
$$

takes $c\left(a x_{1} x_{2}+b x_{3} x_{4}\right)$ to $c\left(x_{1}^{\prime} x_{2}^{\prime}+a b x_{3}^{\prime} x_{4}^{\prime}\right)$. With this, it follows from induction that $\omega \in H^{2}\left(\mathbf{Z}^{6}\right)$ can be put in the form $\omega=a x_{1} x_{2}+b x_{3} x_{4}+c x_{5} x_{6}$ with respect to some basis, with $a|b| c$.

Changing the sign of the basis elements can then ensure that $a, b, c \geq 0$. We can further arrange that the basis change matrix has determinant 1, perhaps at the cost of changing the sign of $c$. The remark at the start of the proof then implies that there is an automorphism $A$ with $A_{*}(\alpha)=\left(a x_{1} x_{2}+b x_{3} x_{4}+c x_{5} x_{6}\right) \cap[T]$, with $a, b \geq 0$ and $a|b| c$.

To show the uniqueness of $a, b$, and $c$ we consider the quotient of $H^{2}\left(\mathbf{Z}^{6}\right) /\langle\omega\rangle$, now viewed as an abelian group. The order of the torsion of the quotient group is $a$. Thus, the value of $a$ depends only on the $\operatorname{Aut}\left(\mathbf{Z}^{6}\right)$ orbit of $\omega$.

Next consider $\omega^{2} \in H^{4}\left(\mathbf{Z}^{6}\right)$. When placed in normal form,

$$
\omega^{2}=2 a b\left(x_{1} x_{2} x_{3} x_{4}+\frac{c}{b} x_{1} x_{2} x_{5} x_{6}+\frac{c}{a} x_{3} x_{4} x_{5} x_{6}\right) .
$$

This shows that $\omega^{2}$ is $2 a b$ times a primitive class in $H^{4}\left(\mathbf{Z}^{6}\right)$. This is a property that is invariant under the action of $\operatorname{Aut}\left(\mathbf{Z}^{6}\right)$ on $H^{4}\left(\mathbf{Z}^{6}\right)$, and so $2 a b$ is uniquely determined. Since $a$ is determined by $\omega$, and $a, b \geq 0, b$ is uniquely determined.

Continuing, $\omega^{3}=6 a b c x_{1} x_{2} x_{3} x_{4} x_{5} x_{6}$. As before this shows the product $6 a b c$ is determined up to sign (since $c$ can be negative) and hence also $|c|$ is determined. The sign of $c$ is also uniquely determined. This is because any determinant 1 automorphism of $\mathbf{Z}^{6}$ fixes the product $6 a b c$ and we require that $a, b \geq 0$.

Thus to identify $\mathcal{G}\left(\mathbf{Z}^{6},[\alpha]\right)$ it suffices to consider $\alpha \in H_{4}\left(\mathbf{Z}^{6}\right)$ with $\alpha=\omega \cap[T]$ and

$$
\omega=a x_{1} x_{2}+b x_{3} x_{4}+c x_{5} x_{6},
$$

where $a|b| c$ and $a, b \geq 0$. Here are the results.

Theorem 5.9. Given $\alpha \in H_{4}\left(\mathbf{Z}^{6}\right)$, there exist unique integers a, $b, c$ satisfying $a|b| c$ with $a, b \geq 0$ and $A \in \operatorname{Aut}\left(\mathbf{Z}^{6}\right)$ so that $A_{*}(\alpha)=\left(a x_{1} x_{2}+b x_{3} x_{4}+c x_{5} x_{6}\right) \cap[T]$. Write $q_{a, b, c}(\sigma)$ for $q_{\mathbf{Z}^{6}, \alpha}(\sigma)$.

Then $q_{a, b,-c}(\sigma)=q_{a, b, c}(-\sigma)$ and the following equalities and estimates hold:

(1) $q_{0,0,0}(\sigma)=20+|\sigma|$.

(2) $q_{1,0,0}(\sigma)=14+|\sigma|$.

(3) $q_{1,1,0}(\sigma)=10+|\sigma|$.

(4)

$$
q_{1,1,1}(\sigma)= \begin{cases}6 & \text { if } \sigma=-2 \text { or } 0 \\ 7 & \text { if } \sigma=-1 \text { or } 1, \\ 4-\sigma & \text { if } \sigma \leq-2, \\ r+\sigma & \text { if } \sigma \geq 2, \text { for some } r \in\{4,6\} .\end{cases}
$$

(5) For general $a, b, c$,

$$
\max \{s, 4+|\sigma|\} \leq q_{a, b, c}(\sigma) \leq s+|\sigma|,
$$

where $s=20$ if $a>1, s=14$ if $a=1$ and $b>1$, and $s=10$ if $a=b=1$ and $c>1$. 
Proof. The first assertion is just a restatement of Theorem [5.8, Replacing $M$ by $-M$ replaces $\alpha$ by $-\alpha$, and hence $q_{a, b, c}(\sigma)=q_{-a,-b,-c}(-\sigma)$. Since we can change the signs of any two of $a, b, c$ by a determinant 1 automorphism of $\mathbf{Z}^{6}$, we see that $q_{-a,-b,-c}(-\sigma)=q_{a, b,-c}(-\sigma)$. Thus we assume $\alpha=\omega \cap[T]$, where $\omega=$ $a x_{1} x_{2}+b x_{3} x_{4}+c x_{5} x_{6}$ with $a, b, c \geq 0$ and $a|b| c$. For convenience we write $\mathcal{M}(G, \omega)$ instead of $\mathcal{M}(G, \omega \cap[T])$.

We next establish some lower bounds. First recall that in Section 5.2.4 we showed that for any $\alpha \in H_{4}\left(\mathbf{Z}^{6}\right), q_{\mathbf{Z}^{6}, \alpha}(\sigma) \geq 2-12+16=6$.

Given $(M, f) \in \mathcal{M}\left(\mathbf{Z}^{6}, \omega\right)$, the 7 -dimensional subspace of $I(M)$,

$$
V_{1}=\left\langle x_{1} x_{2}, x_{1} x_{3}, x_{1} x_{4}, x_{1} x_{5}, x_{3} x_{5}, x_{3} x_{6}, x_{4} x_{5}\right\rangle
$$

is isotropic (we use the map $f^{*}: H^{2}\left(T^{n}\right) \rightarrow H^{2}(M)$ to identify the generators of $H^{2}\left(T^{n}\right)$ with a set of generators of $\left.I(M)\right)$. In fact, the cup product of any two elements in $V_{1}$ with $\omega$ vanishes. Thus the intersection form of $M$ has a 7dimensional isotropic subspace and hence $\beta_{2}(M) \geq 14+|\sigma(M)|$. Hence

$$
q_{a, b, c}(\sigma) \geq \max \{6,4+|\sigma|\}
$$

for any $a, b, c$.

Now suppose that $c=0$. Given $(M, f) \in \mathcal{M}\left(\mathbf{Z}^{6}, a x_{1} x_{2}+b x_{3} x_{4}\right)$, the 10dimensional subspace of $I(M)$,

$$
V_{2}=\left\langle x_{1} x_{2}, x_{1} x_{3}, x_{1} x_{4}, x_{1} x_{5}, x_{1} x_{6}, x_{2} x_{3}, x_{2} x_{4}, x_{3} x_{4}, x_{3} x_{5}, x_{3} x_{6}\right\rangle
$$

is isotropic. Thus

$$
q_{a, b, 0}(\sigma) \geq 10+|\sigma| .
$$

One can get a bit more information from the space $V_{2}$. Suppose that $c>1$. Then if $p$ is a prime which divides $c$, working $\bmod p$ one sees that the $\mathbf{Z} / p$ reduction of $V_{2}$ is isotropic. This allows us to conclude

$$
q_{a, b, c}(\sigma) \geq 10 \text { if }|c|>1 .
$$

Now consider the case $b, c=0$. Given $(M, f) \in \mathcal{M}\left(\mathbf{Z}^{6}, a x_{1} x_{2}\right)$, the 12-dimensional subspace of $I(M)$,

$$
V_{3}=\left\langle x_{1} x_{2}, x_{1} x_{3}, x_{1} x_{4}, x_{1} x_{5}, x_{1} x_{6}, x_{2} x_{3}, x_{2} x_{4}, x_{2} x_{5}, x_{2} x_{6}, x_{3} x_{4}, x_{3} x_{5}, x_{3} x_{6}\right\rangle
$$

is isotropic. Hence

$$
q_{a, 0,0}(\sigma) \geq 14+|\sigma| \text {. }
$$

More generally, if $b>1$, working $\bmod p$ for a prime $p$ dividing $b$ one finds that $V_{3}$ is isotropic, so that

$$
q_{a, b, c}(\sigma) \geq 14 \text { if } b>1 .
$$

Finally, consider the case $a=b=c=0$. For any $(M, f) \in \mathcal{M}\left(\mathbf{Z}^{6}, 0\right)$, the intersection form vanishes on $I(M) \subset H^{2}(M)$, which has rank 15. This implies that

$$
q_{0,0,0}(\sigma) \geq|\sigma|+20 .
$$

As before (or applying Theorem 4.6), one sees

$$
q_{a, b, c}(\sigma) \geq 20 \text { if } a>1 .
$$

Finding good upper bounds is more involved. We begin with a general construction which covers many cases.

Start with $M^{\prime}=\left(F_{2} \times F_{1}\right) \# T^{4}$, where $F_{g}$ denotes the closed oriented surface of genus $g$. Label the generators of $\pi_{1}\left(M^{\prime}\right)$ as follows: $a_{1}, a_{2}, a_{3}, a_{4}$ generate $\pi_{1}\left(F_{2}\right)$, 
$b_{1}, b_{2}$ generate $\pi_{1}\left(F_{1}\right)$, and $c_{1}, c_{2}, c_{3}, c_{4}$ generate $\pi_{1}\left(T^{4}\right)$. Thus the $a_{i}$ commute with the $b_{i}$, the $b_{i}$ commute, the $c_{i}$ commute, and $\left[a_{1}, a_{2}\right]\left[a_{3}, a_{4}\right]=1$.

For $a, b, c$ as above, choose a map $f^{\prime}: M^{\prime} \rightarrow T^{6}$ which induces the map

$$
\begin{aligned}
a_{1} \mapsto \gamma_{1}, a_{2} & \mapsto \gamma_{2}^{b}, a_{3} \mapsto \gamma_{3}, a_{4} \mapsto \gamma_{4}^{a}, \\
b_{1} & \mapsto \gamma_{5}, b_{2} \mapsto \gamma_{6}, \\
c_{1} \mapsto \gamma_{1}^{c}, c_{2} & \mapsto \gamma_{2}, c_{3} \mapsto \gamma_{3}, c_{4} \mapsto \gamma_{4}
\end{aligned}
$$

on fundamental groups. Then $f_{*}^{\prime}\left(\left[M^{\prime}\right]\right)=\left(a x_{1} x_{2}+b x_{3} x_{4}+c x_{5} x_{6}\right) \cap\left[T^{6}\right]$. Note that $\chi\left(M^{\prime}\right)=-2$ and $\sigma\left(M^{\prime}\right)=0$. This is true for any triple $a, b, c$ of integers, regardless of divisibility.

Next perform four surgeries on $M^{\prime}$ to kill the elements $a_{1}^{c} c_{1}^{-1}, a_{2} c_{2}^{-b}, a_{3} c_{3}^{-1}$, and $a_{4} c_{4}^{-a}$. The map to $T^{6}$ extends over the resulting manifold, which we denote by $M^{\prime \prime}$. The fundamental group of $M^{\prime \prime}$ is generated by $a_{1}, c_{2}, c_{3}, c_{4}, b_{1}$, and $b_{2}$, which are mapped to $\gamma_{1}, \gamma_{2}, \cdots, \gamma_{6}$ respectively. Note that $\chi\left(M^{\prime \prime}\right)=6$ and $\sigma\left(M^{\prime \prime}\right)=0$.

For general $a, b, c$, the commutators

$$
\begin{gathered}
z_{1}=\left[a_{1}, c_{2}\right], z_{2}=\left[a_{1}, c_{3}\right], z_{3}=\left[a_{1}, c_{4}\right], z_{4}=\left[c_{2}, b_{1}\right], \\
z_{5}=\left[c_{2}, b_{2}\right], z_{6}=\left[c_{4}, b_{1}\right], z_{7}=\left[c_{4}, b_{2}\right],
\end{gathered}
$$

in $\pi_{1}\left(M^{\prime \prime}\right)$ need not be trivial (but $\left[c_{3}, b_{1}\right]=1=\left[c_{3}, b_{2}\right]$ ). Six surgeries on $M^{\prime \prime}$ to kill these 6 commutators yield $(M, f) \in \mathcal{M}\left(\mathbf{Z}^{6}, \omega\right)$ with $\chi(M)=20$ and $\sigma(M)=0$, for any $a, b, c$. Thus we see that $q_{a, b, c}(\sigma) \leq 20+|\sigma|$ for any $a, b, c$. Combined with the lower bounds derived above we conclude that

$$
q_{0,0,0}(\sigma)=20+|\sigma|
$$

and

$$
q_{a, b, c}(0)=20 \text { for } a>1 .
$$

To treat the case $(a, b, c)=(1, b, c)$, it is convenient instead to realize $\omega=$ $a x_{1} x_{2}+b x_{3} x_{4}+x_{5} x_{6}$, and then to change coordinates. Suppose, then, that $c=1$ and $a, b$ are arbitrary. Then $a_{1}=c_{1}$ in $\pi_{1}\left(M^{\prime \prime}\right)$. Thus $z_{1}=z_{2}=z_{3}=1$ in $\pi_{1}\left(M^{\prime \prime}\right)$. Hence only four surgeries are required to abelianize $\pi_{1}\left(M^{\prime \prime}\right)$, yielding $(M, f) \in \mathcal{M}\left(\mathbf{Z}^{6}, \omega\right)$ with $\chi(M)=14$ and $\sigma(M)=0$. One can find a determinant 1 automorphism that takes $\omega=a x_{1} x_{2}+b x_{3} x_{4}+x_{5} x_{6}$ to $x_{1} x_{2}+b x_{3} x_{4}+a x_{5} x_{6}$. Thus $q_{1, b, c}(\sigma) \leq 14+|\sigma|$. Combined with the lower bounds derived above we conclude that

$$
q_{1,0,0}(\sigma)=14+|\sigma|
$$

and

$$
14 \leq q_{1, b, c}(\sigma) \leq 14+|\sigma|
$$

when $b>1$. We will improve this below when $b=0$.

When $a=1=b$, then in $\pi_{1}\left(M^{\prime \prime}\right),\left[a_{3}, a_{4}\right]=\left[c_{3}, c_{4}\right]=1$, and so $1=\left[a_{1}, a_{2}\right]=$ $\left[a_{1}, c_{2}\right]=z_{1}$. Moreover, $z_{4}=\left[c_{2}, b_{1}\right]=\left[a_{2}, b_{1}\right]=1$ and similarly $z_{5}=1$. Clearly $z_{6}=z_{7}=1$. Thus to abelianize $\pi_{1}\left(M^{\prime \prime}\right)$ requires only two surgeries, yielding $(M, f)$ with $\chi(M)=10$ and $\sigma(M)=0$. This implies that $q_{1,1,0}(\sigma) \leq 10+|\sigma|$ and so combined with the lower bounds one concludes

$$
q_{1,1,0}(\sigma)=10+|\sigma|
$$

and

$$
q_{1,1, c}(0)=10 \text { if } c>1
$$


When $a=b=c=1$, then $\pi_{1}\left(M^{\prime \prime}\right) \cong \mathbf{Z}^{6}$, and so $q_{1,1,1}(\sigma) \leq 6+|\sigma|$ and $q_{1,1,1}(0)=6$. Moreover, the manifold $S_{6}$ has $\chi\left(S_{6}\right)=6$ and $\sigma\left(S_{6}\right)=-2$. It follows from the bounds derived above that if $f: S_{6} \rightarrow B_{\mathbf{Z}^{6}}$ induces an isomorphism on fundamental groups, $f_{*}\left(\left[S_{6}\right]\right)$ corresponds to $a=1, b=1$, and $c= \pm 1$. That $c$ is in fact 1 follows from a symmetry argument, as follows. Notice that there is an automorphism of the genus three surface $F_{3}$ inducing the map on homology carrying the ordered set $\left(x_{1}, x_{2}, \ldots, x_{6}\right)$ of generators of $H_{1}\left(F_{3}\right)$ to $\left(x_{3}, x_{4}, x_{5}, x_{6}, x_{1}, x_{2}\right)$. This induces an orientation preserving homeomorphism of $S_{6}$ inducing a similar map on $\pi_{1}$. Thus, precomposing the map $S_{6} \rightarrow T^{6}$ with this homeomorphism does not affect which class is represented in $H_{4}\left(T^{6}\right)$, but induces a map that carries $a x_{1} x_{2}+b x_{3} x_{4}+c x_{5} x_{6}$ to $b x_{1} x_{2}+c x_{3} x_{4}+a x_{5} x_{6}$. Hence, $a=b=c$.

We now have that $q_{1,1,1}(\sigma) \leq 6+|\sigma+2|$. Thus $q_{1,1,1}(\sigma) \leq 6+|\sigma|$ and $q_{1,1,1}(-2)=$ 6 . These estimates are assembled in the equation

$$
q_{1,1,1}(\sigma)= \begin{cases}6 & \text { if } \sigma=-2 \text { or } 0 \\ 7 & \text { if } \sigma=-1 \text { or } 1 \\ 4-\sigma & \text { if } \sigma \leq-2, \\ r+\sigma & \text { if } \sigma \geq 2, \text { for some } r \in\{4,6\} .\end{cases}
$$

When $a=1, b=c=0$ (that is, $\omega=x_{1} x_{2}$ ), consider $M^{\prime}=T^{4} \# S^{1} \times S^{3} \# S^{1} \times S^{3}$ with fundamental group generated by $a_{1}, a_{2}, a_{3}, a_{4}, b, c$. Choose a map $f^{\prime}: M^{\prime} \rightarrow T^{6}$ which induces the map

$$
a_{1} \mapsto \gamma_{3}, a_{2} \mapsto \gamma_{4}, a_{3} \mapsto \gamma_{5}, a_{4} \mapsto \gamma_{6}, b \mapsto \gamma_{1}, c \mapsto \gamma_{6}
$$

Then it is straightforward to see that $f_{*}^{\prime}\left(\left[M^{\prime}\right]\right)=x_{1} x_{2} \cap[T]$, that $\chi\left(M^{\prime}\right)=-4$, and $\sigma\left(M^{\prime}\right)=0$. Nine surgeries are required to abelianize the fundamental group of $M^{\prime}$; each surgery increases $\chi$ by 2 . This yields $(M, f) \in \mathcal{M}\left(\mathbf{Z}^{6}, x_{1} x_{2}\right)$ with $\chi(M)=14$ and $\sigma(M)=0$. Hence $q_{1,0,0}(\sigma) \leq|\sigma|+14$. Combining this with the lower bound yields

$$
q_{1,0,0}(\sigma)=|\sigma|+14
$$

One consequence of the analysis we carried out for the group $G=\mathbf{Z}^{6}$ is that the behavior of $q_{G}(\sigma)$ and $q_{G, \alpha}(\sigma)$ is different than when $G=\mathbf{Z}^{n}, n<6$ or when $G$ is a 2-or 3-manifold group, since in those cases $q_{G}(\sigma)=k+|\sigma|$ whereas $q_{\mathbf{Z}^{6}}$ has more than one local minimum; in fact $q_{\mathbf{Z}^{6}}(s)<q_{\mathbf{Z}^{6}}(s \pm 1)$ for $s=-2,0,2$. We will investigate this further in the next section.

Calculations and estimates of $q_{\mathbf{Z}^{n}}(\sigma)$ for larger $n$ are possible, but become more difficult. In particular we have not succeeded in finding a normal form for $\omega \in H^{n-4}\left(\mathbf{Z}^{n}\right)$ for $n>6$. However, using the calculations above, the manifolds constructed in [19], the $S_{2 k}$ of Proposition 5.7. Theorem 5.2. and the 7-dimensional isotropic subspace of Equation (5.3), the following calculations are straightforward. We omit the explanations.

(1) $p\left(\mathbf{Z}^{n}\right)=2,0,0,2,0,6,4,2$ for $n=0,1,2,3,4,5,6,7$ respectively.

(2) $0 \leq p\left(\mathbf{Z}^{n}\right) \leq 2-2 n+C(n, 2)+\epsilon_{n}=\frac{n^{2}}{2}-\frac{5 n}{2}+2+\epsilon_{n}$ for all $n$,

(3) $p\left(\mathbf{Z}^{n}\right) \leq 4-\frac{5 n}{2}+C(n, 2)=\frac{n^{2}}{2}-\frac{7 n}{2}+4$ for $n$ even.

\section{Bounds On $p\left(\mathbf{Z}^{n}\right)$}

When we began our work in 19 one of the first objectives was to answer a question asked by Weinberger: is $q\left(\mathbf{Z}^{n}\right)$ asymptotic to $n^{2}$ or $n^{2} / 2$ ? More precisely, 
it followed quickly from [1] that

$$
\frac{n^{2}-5 n+4}{2} \leq q\left(\mathbf{Z}^{n}\right) \leq n^{2}-3 n+2 .
$$

The main result of [19] is that $q\left(\mathbf{Z}^{n}\right)$ is always within 1 of the lower bound for $n \geq 6$. Thus, $\lim _{n \rightarrow \infty} q\left(\mathbf{Z}^{n}\right) / n^{2}=\frac{1}{2}$.

Here we would want to consider the similar question for $p\left(\mathbf{Z}^{n}\right)$. Basic estimates show that for $n \geq 6$,

$$
0 \leq p\left(\mathbf{Z}^{n}\right) \leq \frac{n^{2}-5 n+6}{2} .
$$

The lower bound, first identified in 34] using covering space arguments, follows from the fact that for a manifold $M$ with $\pi_{1}(M) \cong \mathbf{Z}^{n}, \sigma(M) \leq \beta_{2}(M)-2(n-1)$, since, by Theorem 5.2 $H^{2}(M)$ contains an isotropic subspace of dimension $n-1$. The upper bound comes about from the fact that the manifolds constructed in [19] to realize the lower bounds of $q\left(\mathbf{Z}^{n}\right)$ have signature 0 .

The use of the manifold $-S_{2 k}$ introduced in Proposition 5.7 lets us improve the estimate for $p\left(\mathbf{Z}^{n}\right)$ in the case of $n$ even:

$$
0 \leq p\left(\mathbf{Z}^{n}\right) \leq \frac{n^{2}-6 n+8}{2} .
$$

Even with this improvement we see that the upper bound for $p\left(\mathbf{Z}^{n}\right)$ remains asymptotic to $n^{2} / 2$ in the sense that each inequality implies that as $n$ goes to infinity, the quotient of the upper bound and $n^{2}$ is $1 / 2$. We do not know how to identify the actual behavior of $p\left(\mathbf{Z}^{n}\right)$ for large $n$, but can show that it is not asymptotic to the upper bound.

Theorem 6.1. For an infinite set of $n, p\left(\mathbf{Z}^{n}\right) / n^{2} \leq \frac{13}{28}$.

The proof of this result is a delicate construction, efficiently building examples for large $n$ from connected sums of symmetric products of surfaces. The combinatorics is surprisingly best described in terms of projective planes over finite fields, and we begin with a detour into some of the structure of finite projective spaces.

6.1. Points and lines in finite projective space. Let $\mathbf{F}$ denote the finite field with $p$ elements, where $p$ is a prime power. (Our best estimate of $p\left(\mathbf{Z}^{n}\right)$ will come from setting $p=7$.) Let $P^{k}$ denote the $k$-dimensional projective space over $\mathbf{F}$.

\section{Lemma 6.2.}

(1) The number of points in $P^{k}$ is $n=\frac{p^{k+1}-1}{p-1}$.

(2) The number of lines in $P^{k}$ is $L=\frac{\left(p^{k+1}-1\right)\left(p^{k}-1\right)}{(p+1)(p-1)^{2}}$.

Proof. (1) Recall that $P^{k}$ is the quotient of $\mathbf{F}^{k+1}-0$ by a free action of $\mathbf{F}-0$. This gives the number of points in $P^{k}$. Notice that if $k=1$, the projective line $P^{1}$ contains $p+1$ points.

(2) Denote the number of points in $P^{k}$ by $n$. Each pair of distinct points in $P^{k}$ determines a unique line, and thus we get $C(n, 2)$ lines. This count has repetitions, with each line being counted once for each pair of distinct points in that line, and each line has $p+1$ points in it. Thus, the number of lines is $C(n, 2) / C(p+1,2)$. 
Expanding, this can be written as:

$$
\frac{\left(\frac{p^{k+1}-1}{p-1}\right)\left(\frac{p^{k+1}-1}{p-1}-1\right)}{(p+1)(p)} .
$$

Algebraic simplification gives the desired result.

6.2. Building examples. Fix a prime power $p$ and integer $k$, let $n$ be the number of points in $P^{k}$ and let $L$ be the number of lines in $P^{k}$. Let $X$ be a 4 -manifold with $\pi_{1}(X) \cong \mathbf{Z}^{p+1}, \beta_{2}(X)=\beta_{2}, \chi(X)=\chi$, and $\sigma(X)=\sigma$.

For each line $l \subset P^{k}$, let $X_{l}$ denote a copy of $X$. We will index the generators of $\pi_{1}\left(X_{l}\right)$ with the points of $l$, in arbitrary order. Let $Y$ be the connected sum of all the $X_{l}$.

Now, perform surgeries on $Y$ to identify certain pairs of generators: if a generator of $X_{l_{1}}$ and a generator of $X_{l_{2}}$ are indexed with the same point of $P^{k}$, use a surgery to identify these. Call the resulting manifold $W$.

Theorem 6.3. $\pi_{1}(W) \cong \mathbf{Z}^{n}, \beta_{2}(W)=L \beta_{2}$, and $\sigma(W)=L \sigma$.

Proof. Clearly $\pi_{1}(W)$ has $n$ generators, and the first homology is $\mathbf{Z}^{n}$. We claim that $\pi_{1}(W)$ is abelian. Given any two generators, they are indexed by two points in $P^{k}$. These two points determine a line $l$ in $P^{k}$. Thus, the generators commute, because they both have representatives on $X_{l}$, which has an abelian fundamental group.

Since signature adds under connected sum and surgery does not change the signature, we have $\sigma(W)=L \sigma(X)$, as desired.

Since $Y$ is a connected sum, $\beta_{2}(Y)=L \beta_{2}(X)$. Each surgery is along a curve that is of infinite order in homology, so the surgeries do not change the second Betti number: $\beta_{2}(W)=L \beta_{2}$.

Our goal is to attain asymptotics for an upper bound for $p\left(\mathbf{Z}^{n}\right)$. As our manifold $X$ we use $X=-S_{p+1}$ (see Proposition 5.7) so that $\pi_{1}(X)=\mathbf{Z}^{p+1}, \beta_{2}(X)=$ $\left(p^{2}+p+2\right) / 2, \sigma(X)=(p-1) / 2$. Noting that $p\left(\mathbf{Z}^{n}\right) \leq \chi(W)-\sigma(W) \leq \beta_{2}(W)-$ $\sigma(W)$, we compute

$$
\beta_{2}(W)-\sigma(W)=L\left(\frac{p^{2}+3}{2}\right) .
$$

Dividing by $n^{2}$ this upper bound simplifies to be $\frac{p^{2}+3}{2\left(p^{2}+p\right)}$ when terms that go to zero as $k$ increases are removed. An elementary calculus exercise applies to show that the minimum of this function among primes occurs at either $p=5$ or $p=7$, and then a calculation shows the minimum occurs at $p=7$, where the limit is $\frac{13}{28}$. This completes the proof of Theorem 6.1.

\subsection{Local minima for $q_{\mathbf{Z}^{n}}(\sigma)$. Let}

$$
C=\{(k-\ell, k+\ell) \mid k, \ell \text { nonnegative integers }\} .
$$

Thus $C$ consists of all integer lattice points in the $(x, y)$-plane whose coordinates have the same parity and which satisfy $y \geq|x|$.

From Corollary 3.2 we know that $\mathcal{G}(G)$ is a union of cones of the type

$$
C_{a, b}=\{(a, b)\}+C
$$

since if $(a, b) \in \mathcal{G}(G)$, so is $(a, b)+(c, d)$ for any $(c, d) \in C$. 
Definition 6.4. A positive integer $a$ is called a minimum point of $q_{G}$ if $q_{G}$ has a local minimum at $a$, i.e. $q_{G}(a+1)=q_{G}(a-1)=q_{G}(a)+1$.

The minimum points determine the geography completely, and correspond to irreducible manifolds when $G$ is not a free product, as the following theorem shows.

Theorem 6.5. For any $G, q_{G}$ has finitely many minimum points. Moreover, $\mathcal{G}(G)$ is the union of the cones $C_{a, q_{G}(a)}$ as a runs over the minimum points.

If a 4-manifold $M \in \mathcal{M}(G)$ represents a minimum point a (in other words, if $\left.(\sigma(M), \chi(M))=\left(a, q_{G}(a)\right)\right)$, and if $M$ is homotopy equivalent to a connected sum $N \# X$ for a 4-manifold $N$ and a simply connected 4-manifold $X$, then $X$ is homeomorphic to the 4-sphere. In particular, if $G$ is not a free product, then $M$ is irreducible as a topological 4-manifold.

Proof. The function $q_{G}(\sigma)-\sigma$ is integer valued and decreasing. As $\sigma \rightarrow \infty$, Theorem 3.4. Part 6 implies that $q_{G}(\sigma)-\sigma$ is bounded below by $p(G)$. Thus there are finitely many $a \geq 0$ so that $q_{G}(a)-a<q_{G}(a-1)-(a-1)$. Since any minimum point $a$ with $a>0$ satisfies $q_{G}(a-1)=q_{G}(a)+1$, it follows that there are finitely many minimum points $a$ with $a \geq 0$. A similar argument using $q_{G}(\sigma)+\sigma$ shows that there are finitely many negative minimum points.

If $\left(x, q_{G}(x)\right)$ is in a $C_{a, q_{G}(a)}$ for some minimum point $a$, then so is $(x, y)$ for any $y>q_{G}(x), y \equiv x \bmod 2$; hence to prove the second assertion it suffices to show that for each integer $x,\left(x, q_{G}(x)\right)$ lies in $C_{a, q_{G}(a)}$ for some minimum point $a$. If $x$ is a minimum point, the conclusion is obvious. If $q_{G}(x+1)<q_{G}(x)$, then since $q_{G}$ is bounded below by $q(G)$ and since $q_{G}(\sigma+1)=q_{G}(\sigma) \pm 1$ for all $\sigma$ (Theorem 3.4. Parts 4 and 7) there is an integer $z$ greater than $x$ so that $q_{G}$ has a local minimum at $z$. If $a$ denotes the least integer greater than $x$ so that $q_{G}$ has a local minimum at $a$, then clearly $\left(x, q_{G}(x)\right) \in C_{a, q_{G}(a)}$. A similar argument applies if $q_{G}(x-1)<q_{G}(x)$.

Let $M \in \mathcal{M}(G)$ satisfy $(\sigma(M), \chi(M))=\left(a, q_{G}(a)\right)$ for some $a$. If $M$ is homotopy equivalent to $N \# X$ where $X$ is simply connected, then $\chi(N)=\chi(M)-\beta_{2}(X)$ and $\sigma(N)=\sigma(M)-\sigma(X)$.

Let $k=\sigma(X)$. If $k=0$, then $\sigma(N)=\sigma(X)$, and so $\chi(N) \geq \chi(M)$, since $\chi(M)=q_{G}(a)$. Thus $\beta_{2}(X)=0$ and so $X$ is a homotopy 4 -sphere, and so by the 4-dimensional topological Poincaré conjecture ([6]), $X$ is homeomorphic to $S^{4}$.

If $k>0$, then $N \#_{k-1} \mathbf{C P}^{2}$ has signature equal to $\sigma(M)-1$ and Euler characteristic equal to $\chi(M)-\beta_{2}(X)+(k-1) \leq \chi(M)-1=q_{G}(a)-1$. Thus $q_{G}(a-1) \leq q_{G}(a)-1$ and so $a$ is not a minimum point. A similar argument using $-\mathbf{C P}^{2}$ shows that if $k<0$, then $a$ is not a minimum point.

One can make the same definition of minimum points for $q_{G, \alpha}$ for any $\alpha \in H_{4}(G)$. The assertions of Theorem 6.5 extend with the same proofs. Note that for $q_{G}$ (but not necessarily $q_{G, \alpha}$ ) the minimum points are symmetric with respect to 0 , as one sees by reversing orientation. Clearly the main challenge of understanding the geography problem for a group $G$ is to identify all the minimum points (and find the corresponding manifolds) of $q_{G}$ (and of $q_{G, \alpha}$ ).

For example, for $G$ any 2- or 3-manifold group, or $\mathbf{Z}^{n}$ when $n<6, a=0$ is the only minimum point. For $G=\mathbf{Z}^{6}$, the minimum points are exactly $-2,0$, and 2 . For any $\mathbf{Z}^{n}, 0$ is a minimum point.

The construction of Theorem 6.3 gives groups with at least 5 minimum points. For example, taking $G=\mathbf{Z}^{156}$, the example from [19] has $\sigma=0$ and $\chi=11780$. 
The manifold $-S_{156}$ of Proposition 5.7 has $\sigma=77$ and $\chi=11781$. The construction of Theorem 6.3 taking $p=5$ and $k=3$ yields a manifold with fundamental group $\mathbf{Z}^{156}, \sigma=1612$ and $\chi=12586$. It follows that $q_{\mathbf{Z}^{156}}$ has at least two positive minimum points ( $a=77$ is a minimum point, and there is at least one minimum point $a$ satisfying $79 \leq a \leq 2408)$, and from symmetry that $q_{\mathbf{Z}^{156}}$ has at least five minimum points.

A variation of the argument of Theorem 6.3 shows how to construct examples with many minimum points. We show next that $q_{\mathbf{Z}^{57}}$ has at least 13 minimum points. We leave to the reader the exercise of showing that for any $N$ there exists an $n$ so that $q_{\mathbf{Z}^{n}}$ has at least $N$ minimum points.

In [19] a manifold $C_{8}$ with fundamental group $\mathbf{Z}^{8}$, second Betti number $\beta_{2}=28$, and signature $\sigma=0$ is constructed. Take $p=7$ and $k=2$ in Theorem 6.3, so that $n=57$ and $L=57$. For each $s=0,1,2, \ldots, 57$, construct the manifold $W_{s}$ similarly to the construction of $W$ but starting with the connected sum of $s$ copies of $-S_{8}$ and $57-s$ copies of $C_{8}$ and surgering to identify generators indexed by the same point in $P^{3}$. Then $\pi_{1}\left(W_{s}\right)=\mathbf{Z}^{57}, \beta_{2}\left(W_{s}\right)=s 29+(57-s) 28=1,568+s$, and $\sigma\left(W_{s}\right)=3 s$. Hence $\chi\left(W_{s}\right)=1,484+s$.

A detailed calculation shows that the presence of the points $(3 s, 1484+s), s=$ $0,1, \ldots, 57$, in the geography implies that there are at least 6 positive minimum points for the function $q_{\mathbf{Z}^{57}}(\sigma)$. By symmetry, there are at least 13 minimum points in total.

\section{Problems}

(1) Are there groups for which the geography $\mathcal{G}(G)$ depends on the choice of the category of spaces: smooth 4-manifolds, topological 4-manifolds, and 4-dimensional Poincaré duality spaces? We ask a similar question for $\mathcal{G}(G, \alpha), \alpha \in H_{4}(G)$. As a specific example, we have that for $G \cong \mathbf{Z}^{8}$, $\chi \geq 14$, and if $\chi=14$, then the intersection form on $H_{2}(M) \cong \mathbf{Z}^{28}$ is even with $\sigma \leq 14$. Since even intersection forms have signature congruent to $0 \bmod 8$, the only possibilities are $\sigma=0, \pm 8$. By Rochlin's theorem the case $\sigma= \pm 8$ cannot occur in the smooth category. We do not know if $(14, \pm 8) \in \mathcal{G}\left(\mathbf{Z}^{8}\right)$ in the topological category.

(2) Develop techniques specific to the smooth category to analyze $\mathcal{G}(G)$. Formulate the 4-dimensional topological surgery theory needed to relate the problems of computing $\mathcal{G}(G)$ in the categories of topological 4-manifolds and 4-dimensional Poincaré complexes.

(3) For nontrivial classes $\alpha \in H_{4}(G)$, find relationships between $\mathcal{G}(G, \alpha)$ and $\mathcal{G}(G, k \alpha), k>1$. For instance, if $M \in \mathcal{M}(G, \alpha)$ has a connected $k$-fold covering space, then one can construct an $M^{\prime} \in \mathcal{M}(G, k \alpha)$ in a fairly natural way, and often this is more efficient than starting with the connected sum of $k$ copies of $M$. For $G \cong \mathbf{Z}^{n}$ this yields the best examples we know.

(4) It seems unlikely that $\mathcal{G}(G, \alpha)$ is always symmetric with respect to reflection through the $\chi$-axis when $\alpha$ is nontrivial. Find a counterexample. Is $\mathcal{G}\left(\mathbf{Z}^{6},\left(x_{1} x_{2}+x_{3} x_{4}+x_{5} x_{6}\right) \cap[T]\right)$ symmetric?

(5) Find relations between properties of a group $G$ and the geography of $G$. As an example, according to 23, the methods of $L^{2}$-homology show that if $G$ is amenable, $q(G) \geq 0$ and $p(G) \geq 0$. 
(6) Find a finitely presented group $G$ which is not the fundamental group of any Poincaré 3-complex (finite or infinite), $G$ is not the free product with a free group, $H_{4}(G)=0$, and $q_{G}(\sigma)=2+|\sigma|$.

(7) How is the geography of a free product, $\mathcal{G}\left(G_{1} * G_{2}\right)$, related to $\mathcal{G}\left(G_{1}\right)$ and $\mathcal{G}\left(G_{2}\right)$ ? (See [20] for an early discussion of this question.) Using connected sums one has $q\left(G_{1} * G_{2}\right) \leq q\left(G_{1}\right)+q\left(G_{2}\right)-2$. Note, however, that according to 22 the inequality can be strict: if $p_{0}$ and $p_{1}$ are distinct primes, then $q\left(\mathbf{Z} / p_{i} \times \mathbf{Z} / p_{i}\right)=4$ as follows from Propositions 4.1 and 4.6 . On the other hand, $q\left(\left(\mathbf{Z} / p_{0} \times \mathbf{Z} / p_{0}\right) *\left(\mathbf{Z} / p_{1} \times \mathbf{Z} / p_{1}\right)\right)=4$, since $\left(\mathbf{Z} / p_{0} \times \mathbf{Z} / p_{0}\right) *$ $\left(\mathbf{Z} / p_{1} \times \mathbf{Z} / p_{1}\right)$ has a presentation with 4 generators but only 5 relations instead of the expected 6 (see the original source, [15], or [22]).

For free abelian groups similar examples are not known, but seem possible. For instance, we have $q\left(\mathbf{Z}^{6}\right)=6$ (see Section 5) but do not know whether $q\left(\mathbf{Z}^{6} * \mathbf{Z}^{6}\right)=9$ or 10 . The predicted value using additivity would be 10 .

(8) A class $\omega \in H^{n-4}\left(\mathbf{Z}^{n}\right)$ determines an even symmetric bilinear form

$$
\phi: H^{2}\left(\mathbf{Z}^{n}\right) \times H^{2}\left(\mathbf{Z}^{n}\right) \rightarrow \mathbf{Z}
$$

by $x y \omega=\phi(x, y)[T]$, where $[T]$ is a chosen generator of $H^{n}\left(\mathbf{Z}^{n}\right)$. What unimodular forms can arise in this way? The first unresolved case, mentioned in the first problem, is for $n=8$, where $H^{2}\left(\mathbf{Z}^{8}\right) \cong \mathbf{Z}^{28}$ and we do not know whether the form $10 H \oplus E_{8}$ can occur.

(9) Suppose $\omega \in H^{n-4}\left(\mathbf{Z}^{n}\right)$ determines a unimodular form $\phi$. Does there exist a smooth or topological 4-manifold with intersection form $\phi$ ? Does there exist a 4-dimensional Poincaré complex with intersection form $\phi$ ?

(10) Determine the asymptotic behavior of the geography of $\mathbf{Z}^{n}$ in terms of $n$. For instance, the main result of [19] is that $q\left(\mathbf{Z}^{n}\right)$ is roughly asymptotic to $n^{2} / 2$. It follows that $p\left(\mathbf{Z}^{n}\right)$ is also bounded above (asymptotically) by $n^{2} / 2$, but according to Theorem 6.1 this is not the best possible: there are arbitrarily large $n$ for which $p\left(\mathbf{Z}^{n}\right) \leq \frac{13}{28} n^{2}$. Is it possible that $p\left(\mathbf{Z}^{n}\right) / n^{2}$ goes to 0 for large $n$ ?

(11) Determine $\mathcal{G}\left(\mathbf{Z}^{n}, \alpha\right)$ for some $n$ and nonprimitive class $\alpha \in H_{4}\left(\mathbf{Z}^{n}\right)$. The first case is $\mathcal{G}\left(\mathbf{Z}^{4}, 2[T]\right)$, where $[T]$ is a generator of $H_{4}\left(\mathbf{Z}^{4}\right) \cong \mathbf{Z}$.

(12) For a given $N$ find a group $G$ and $\alpha \in H_{4}(G)$ so that $q_{G, \alpha}$ has at least $N$ minimum points. We do not know an example for $N=2$.

\section{ACKNOWLEDGMENTS}

The authors would like to thank P. Teichner, I. Hambleton, and C. Van Cott for many helpful comments, and M. Larsen for providing insights into our study of the asymptotics related to Kotschick's function $p(G)$ in Section 6.1.

\section{REFERENCES}

1. S. Baldridge and P. Kirk, On symplectic 4-manifolds with prescribed fundamental group, Commentarii Math. Helv. 82 (2007) 845-875. MR2341842

2. H. Cartan and S. Eilenberg, Homological Algebra, Princeton, 1956. MR0077480 (17:1040e)

3. J. Cheeger and M. Gromov, $L^{2}$-cohomology and group cohomology, Topology 25 (1986) 189215. MR837621 (87i:58161)

4. B. Eckmann, 4-manifolds, group invariants, and $l_{2}-$ Betti numbers, Enseign. Math. (2) 43 (1997), no. 3-4, 271-279. MR.1489886 (99b:57002) 
5. B. Eckmann, Introduction to $l_{2}$-methods in topology: Reduced $l_{2}$-homology, harmonic chains, $l_{2}-$ Betti numbers, Notes prepared by Guido Mislin, Israel J. Math. 117 (2000), 183-219. MR 1760592 (2001b:57054)

6. M. Freedman and F. Quinn, 'Topology of 4-manifolds,' Princeton Mathematical Series, 39, Princeton University Press, Princeton, NJ, 1990. MR.1201584 (94b:57021)

7. R. Gompf, A new construction of symplectic manifolds, Ann. of Math. (2) 142 (1995), no. 3, 527-595. MR 1356781 (96j:57025)

8. M. Gromov, Volume and bounded cohomology, Inst. Hautes Etudes Sci. Publ. Math. No. 56 (1982), 5-99. MR686042 (84h:53053)

9. I. Hambleton and M. Kreck, On the classification of topological 4-manifolds with finite fundamental group. Math. Ann. 280 (1988), no. 1, 85-104. MR928299 (89g:57020)

10. I. Hambleton, M. Kreck, and P. Teichner, Nonorientable 4-manifolds with fundamental group of order 2, Trans. Amer. Math. Soc. 344 (1994), no. 2, 649-665. MR1234481 (94k:57031)

11. J.-C. Hausmann and S. Weinberger, Caractéristiques d'Euler et groupes fondamentaux des variétés de dimension 4, Comment. Math. Helv. 60 (1985), 139-144. MR787667(86m:57020)

12. J. Hempel, '3-Manifolds', Ann. of Math. Studies, No. 86, Princeton University Press, Princeton, N. J.; University of Tokyo Press, Tokyo, 1976. MR0415619 (54:3702)

13. J. Hillman, 'The algebraic characterization of geometric 4-manifolds,' London Mathematical Society Lecture Note Series, 198, Cambridge University Press, Cambridge, 1994. MR1275829 (95m:57032)

14. J. Hillman, A homology 4-sphere group with negative deficiency, Enseign. Math. (2) 48 (2002), 259-262. MR 1955602 (2003m:57046)

15. C. Hog, M. Lustig, and W. Metzler, Presentation classes, 3-manifolds and free products, Geometry and Topology (College Park, Md., 1983/84), 154-167, Lecture Notes in Math., 1167, Springer, Berlin, 1985. MR827268 (87g:57005)

16. F. Johnson and D. Kotschick, On the signature and Euler characteristic of certain fourmanifolds, Math. Proc. Cambridge Philos. Soc. 114 (1993), no. 3, 431-437. MR1235990 (94i:57043)

17. R. Kirby, Problems in low-dimensional topology, Edited by Rob Kirby, AMS/IP Stud. Adv. Math., 2.2, Geometric topology (Athens, GA, 1993), 35-473, Amer. Math. Soc., Providence, RI, 1997. MR1470751

18. R. Kirby and L. Siebenmann, 'Foundational essays on topological manifolds, smoothings, and triangulations,' With notes by John Milnor and Michael Atiyah, Annals of Mathematics Studies, No. 88. Princeton University Press, Princeton, N.J.; University of Tokyo Press, Tokyo, 1977. MR.0645390(58:31082)

19. P. Kirk and C. Livingston, The Hausmann-Weinberger 4-manifold invariant of abelian groups, Proc. Amer. Math. Soc. 133 (2005), no. 5, 1537-1546. MR2111955 (2006g:57001)

20. D. Kotschick, Four-manifold invariants of finitely presentable groups, in Topology, Geometry and Field Theory, 89-99, World Sci. Publishing, River Edge, NJ, 1994. MR1312175 (95m:57003)

21. D. Kotschick, Minimizing Euler characteristics of symplectic four-manifolds, Proc. Amer. Math. Soc. 134 (2006), no. 10, 3081-3083. MR2231635(2007d:57041)

22. M. Kreck, W. Lück, and P. Teichner, Counterexamples to the Kneser conjecture in dimension four, Comment. Math. Helv. 70 (1995), no. 3, 423-433. MR.1340102 (96d:57020)

23. W. Lück, $L^{2}$-Betti numbers of mapping tori and groups, Topology 33 (1994), no. 2, 203-214. MR.1273782 (95g:58235)

24. I. G. MacDonald, Symmetric products of an algebraic curve, Topology 1 (1962), 319-343. MR0151460 (27:1445)

25. J. Milnor, Groups which act on $S^{n}$ without fixed points, Amer. J. Math. 79 (1957), 623-630. MR0090056 (19:761d)

26. J. Milnor and D. Husemoller, 'Symmetric bilinear forms,' Ergebnisse der Mathematik und ihrer Grenzgebiete, Band 73. Springer-Verlag, New York-Heidelberg, 1973. MR0506372 (58:22129)

27. J. Milnor and J. Stasheff, 'Characteristic classes,' Annals of Mathematics Studies, No. 76. Princeton University Press, Princeton, N. J. 1974. MR0440554 (55:13428)

28. R. Stong, 'Notes on cobordism theory,' Mathematical notes, Princeton University Press, Princeton, N.J.; University of Tokyo Press, Tokyo 1968. MR0248858 (40:2108)

29. R. Swan, Periodic resolutions for finite groups, Ann. of Math. (2) 72 (1960), 267-291. MR0124895 (23:A2205) 
30. R. Thom, Quelques propriétés globales des variétés différentiables, Comment. Math. Helv. 28, (1954), 17-86. MR0061823(15:890a)

31. C. T. C. Wall, 'Surgery on Compact Manifolds,' Edited and with a foreword by A. A. Ranicki, Mathematical Surveys and Monographs, 69, American Mathematical Society, Providence, RI, 1999. MR 1687388(2000a:57089)

32. G. Whitehead, 'Elements of homotopy theory,' Graduate Texts in Mathematics, 61, SpringerVerlag, New York-Berlin, 1978. MR 516508 (80b:55001)

33. J. H. C. Whitehead, On simply connected, 4-dimensional polyhedra, Comment. Math. Helv. 22 (1949), 48-92. MR0029171 (10:559d)

34. H. E. Winkelnkemper, A theorem on manifolds of dimension 4, Acta Mexicana Ci. Tecn. 2 (1968) 88-89. MR0238347 (38:6623)

Department of Mathematics, Indiana University, Bloomington, Indiana 47405

E-mail address: pkirk@indiana.edu

Department of Mathematics, Indiana University, Bloomington, Indiana 47405

E-mail address: livingst@indiana.edu 